\title{
Comparative chemistry of diffuse clouds. IV: $\mathrm{CH}$
}

\author{
H. Liszt ${ }^{1}$ and R. Lucas ${ }^{2}$ \\ 1 National Radio Astronomy Observatory, 520 Edgemont Road, Charlottesville, VA 22903-2475, USA \\ 2 Institut de Radioastronomie Millimétrique, 300 Rue de la Piscine, 38406 Saint Martin d'Hères, France
}

Received 21 March 2002 / Accepted 6 June 2002

\begin{abstract}
We observed the $3335 \mathrm{MHz}(\lambda 9 \mathrm{~cm}) F=1-1$ line of $\mathrm{CH}$ toward a sample of diffuse clouds occulting compact extragalactic mm-wave continuum sources, using the old NRAO $43 \mathrm{~m}$ telescope. Because radiofrequency observations of $\mathrm{CH}$ really must be calibrated with reference to a known $\mathrm{CH}$ abundance, we begin by deriving the relationships between $\mathrm{CH}, E_{B-V}, \mathrm{H}_{2}$ and other hydrides found by optical spectroscopy. No simple relationship exists between $N(\mathrm{CH})$ and $E_{B-V}$, since $N(\mathrm{CH})$ is strongly bimodal with respect to reddening for $E_{B-V}<0.3$ mag and the typical range in the $N(\mathrm{CH}) / E_{B-V}$ ratio is an order of magnitude or more at any given $E_{B-V}>0.3 \mathrm{mag}$. However, $N(\mathrm{CH}) / N\left(\mathrm{H}_{2}\right)=4.3 \pm 1.9 \times 10^{-8}$ in the mean and $N(\mathrm{CH}) \propto N\left(\mathrm{H}_{2}\right)^{1.00 \pm 0.06}$ for $10^{19}<N\left(\mathrm{H}_{2}\right)<10^{21} \mathrm{~cm}^{-2}$. If $\mathrm{CH}$ is a good predictor of $\mathrm{H}_{2}, 40 \%-45 \%$ of the hydrogen in the local diffuse/translucent ISM is in the molecular form at the accepted mean density, higher than previous estimates found in samples of lower-than-average mean density. Optical observations of the population ratios in the upper and lower halves of the $\mathrm{CH}$ lambda-doublet suggest that the brightness of the $3335 \mathrm{MHz} \mathrm{CH}$ line should be double-valued at a given $\mathrm{CH}$ column density in diffuse gas: double-valuedness is noticeable in our data when comparing $\mathrm{CH}$ with $\mathrm{CO}$ or $\mathrm{HCO}^{+}$. The $\mathrm{CH}$ brightness at $3335 \mathrm{MHz}$ is mildly bimodal with respect to $\mathrm{CO}$ emission in our diffuse cloud data but much more strongly bimodal when comparing diffuse or translucent gas and dark gas. The $\mathrm{CH} \Lambda$-doublet is generally inverted in diffuse gas but we did not succeed in measuring the excitation temperature except toward $3 \mathrm{C} 123$ where we confirm one older value $T_{\mathrm{exc}} \approx-10 \mathrm{~K}$.
\end{abstract}

Key words. ISM: molecules

\section{Introduction}

Some chemical species present in diffuse interstellar gas can only be studied in optical/uv absorption: these include $\mathrm{C}_{2}$ (Chaffee \& Lutz 1978), NH (Meyer \& Roth 1991; Crawford $\&$ Williams 1997), HCl (Federman et al. 1995), and most importantly $\mathrm{H}_{2}$ (Savage et al. 1977). Many more, chiefly polyatomics and heavy diatomics, are seen only in the radio regime. These include $\mathrm{H}_{2} \mathrm{CO}$ (Federman \& Willson 1982; Liszt \& Lucas 1995), $\mathrm{C}_{3} \mathrm{H}_{2}$ (Cox et al. 1988; Lucas \& Liszt 2000a), $\mathrm{NH}_{3}$ (Nash 1990), $\mathrm{HCO}^{+}$(Liszt \& Lucas 1996, 2000), $\mathrm{C}_{2} \mathrm{H}$ (Lucas \& Liszt 2000a), HCN and HNC (Liszt \& Lucas 2001), $\mathrm{SiO}$ (Lucas \& Liszt 2000b), and sulfur-bearing species such as $\mathrm{CS}, \mathrm{SO}, \mathrm{H}_{2} \mathrm{~S}, \mathrm{HCS}^{+}$etc. (Lucas \& Liszt 2002).

The few species which overlap both domains $(\mathrm{CH}, \mathrm{OH}, \mathrm{CN}$ and $\mathrm{CO}$ ) provide an important bridge between two rather different ways of studying interstellar chemistry in diffuse clouds. $\mathrm{CN}$, for instance, whose relationships with $\mathrm{CH}, \mathrm{C}_{2}, \mathrm{CO}$ and $\mathrm{H}_{2}$ are well-studied optically (Federman et al. 1994; van Dishoeck \& Black 1989; Federman \& Lambert 1988), is closely tied to $\mathrm{HCN}$ and HNC and more loosely related to perhaps a dozen other polyatomic species seen at mm-wavelengths (Liszt \& Lucas 2001). Similarly $\mathrm{OH}$, whose abundance relative to $\mathrm{H}_{2}$ was found by Crutcher (1979) to be constant over a wide range of extinction, is closely tied to $\mathrm{HCO}^{+}$(Liszt \& Lucas 1996;

Send offprint requests to: H. S. Liszt, e-mail: hliszt@nrao.edu
Lucas \& Liszt 1996; Liszt \& Lucas 2000), leading us to believe that the relative abundance of $\mathrm{HCO}^{+}$also varies little. $\mathrm{HCO}^{+}$ is the immediate progenitor of $\mathrm{CO}$ in diffuse gas (Glassgold \& Langer 1975, 1976; Van Dishoeck \& Black 1986). Its unexpectedly large abundance relative to $\mathrm{H}_{2}$ is sufficient to explain the run of $N(\mathrm{CO})$ with $N\left(\mathrm{H}_{2}\right)$ for $2 \times 10^{19}<N\left(\mathrm{H}_{2}\right)<2 \times 10^{21} \mathrm{~cm}^{-2}$. $4 \times 10^{12}<N(\mathrm{CO})<2 \times 10^{16} \mathrm{~cm}^{-2}$ (Liszt \& Lucas 2000).

$\mathrm{CH}$, the subject of this study, represents another important bridge between the optical and radio regimes, all the more so because its abundance relative to molecular hydrogen is very well-determined and nearly fixed for lines of sight having $A_{V} \leq 3$ (Federman \& Lambert 1988; Danks et al. 1984; Federman 1982). A determination of its column density in the gas toward our compact extragalactic mm-wave continuum background sources would provide an important check on the abundances of all the other species we detect. Unfortunately, observation of $\mathrm{CH}$ at $3335 \mathrm{MHz}$ in the radio regime is presently impossible and opportunities will be at best severely restricted in the future. Of the large single dishes, only Arecibo and Nançay apparently have current plans to provide a receiver in the $9 \mathrm{~cm}$ waveband. Eventually, the complete frequency coverage of the EVLA will make possible widespread study of $\mathrm{CH}$ in stimulated emission against continuum sources, although the usefulness of such work, absent complementary emission studies, may be somewhat limited. 

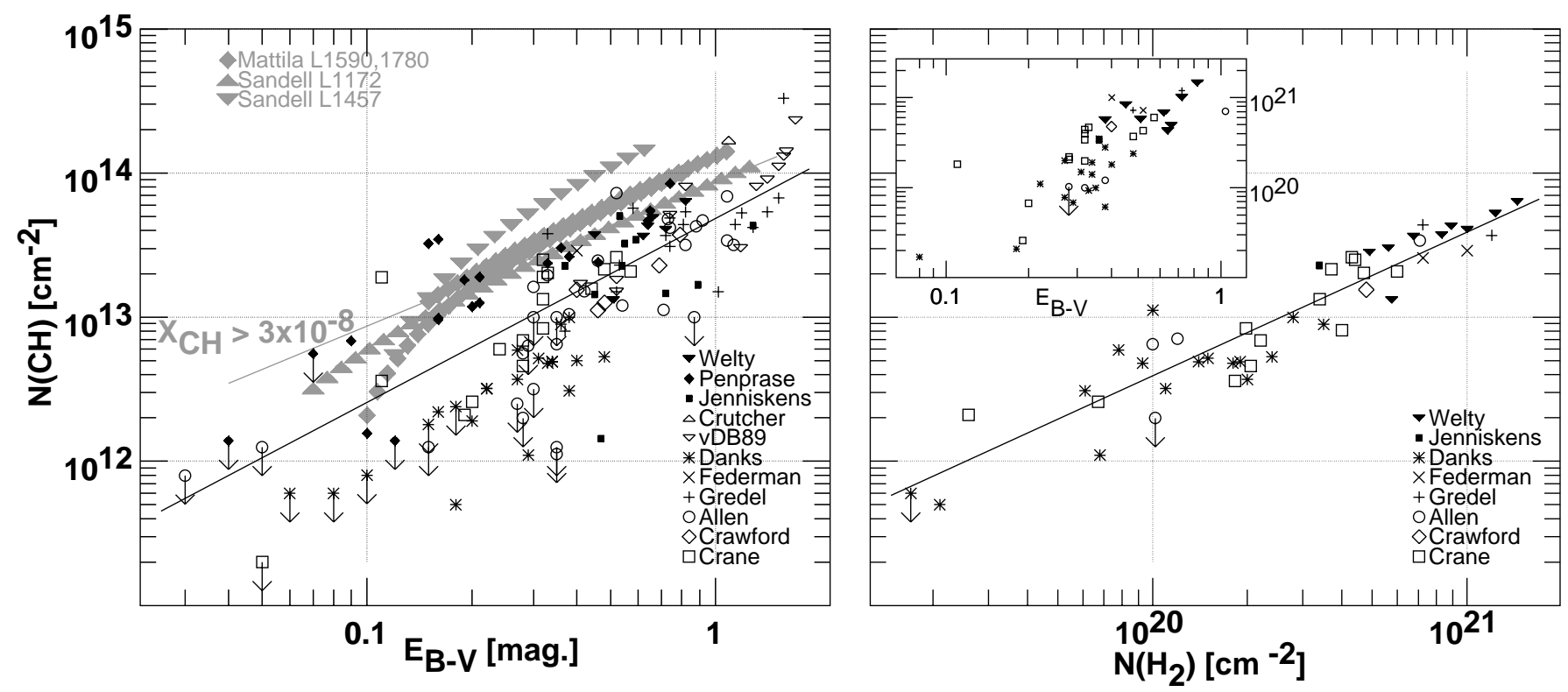

Fig. 1. The dependence of optically-measured $\mathrm{CH}$ column densities on reddening (left panel) and $\mathrm{N}\left(\mathrm{H}_{2}\right)$. $\mathrm{CH}$ Data are from Crane et al. (1995), Crawford (1995), Allen (1994), Gredel et al. (1993), Federman et al. (1994), Danks et al. (1984), van Dishoeck \& Black (1989), Crutcher (1985), Jenniskens et al. (1992), Penprase (1993) and Welty et al. as tabulated in Rachford et al. (2002).

The $\mathrm{H}_{2}$ column densities are from Savage et al. (1977) and Rachford et al. (2002) except for one line of sight each from Joseph et al. (1986), Snow et al. (2000) and Rachford et al. (2001). Superposed in the left panel are the $N(\mathrm{CH})-E_{B-V}$ loci derived from the dark cloud studies of Mattila (1986) for L1590 and L1780, and Sandell (1982) for L1172 and L1457; also shown there is the line corresponding to $X_{\mathrm{CH}} \approx$ $2 N(\mathrm{CH}) / N(\mathrm{H})=3 \times 10^{-8}$ as discussed in the text. Superposed in the right-hand panel are a plot of $N\left(\mathrm{H}_{2}\right)$ vs. $E_{B-V}$ for sightlines with measured $N(\mathrm{CH})$ and the best-fit power-law regression line (see Sect. 2.2).

In this work we present $3335 \mathrm{MHz} F=1-1 \mathrm{CH}$ observations in the direction of compact extragalactic continuum sources which we have previously observed in an extensive, ongoing study of mm-wave absorption line chemistry. We took data toward and around these and two other sources which have stronger $\mathrm{cm}$-wave continuum and are occulted by denser (but not necessarily darker) gas (i.e. 3C123 and 3C133), in much the same way as we did earlier for OH (Liszt \& Lucas 1996; Lucas \& Liszt 1996) and $\mathrm{H}_{2} \mathrm{CO}$ (Liszt \& Lucas 1995). We did so at least partly for the purpose of deriving the excitation temperature in diffuse gas and did not see any true $\mathrm{CH}$ absorption: the optical depth was either negative, owing to the well-known collisionally-induced inversion of the ground-state lambda-doublet (Bertojo et al. 1976; Bujarrabal et al. 1984; Bouloy et al. 1984), or immeasurably small. The $\mathrm{CH}$ transition is almost certainly inverted in the diffuse gas we observed but excitation studies will require a telescope gain much larger and a telescope beam much smaller than that afforded by the old NRAO $43 \mathrm{~m}$ antenna. In the absence of much direct evidence allowing a calibration of the relationship between $N(\mathrm{CH})$ and the microwave brightness in diffuse gas, we resort to a discussion of the rich body of optical $\mathrm{CH}$ measurements in the diffuse ISM.

The plan of the present paper is as follows. In Sect. 2 we derive the abundance of $\mathrm{CH}$ with respect to $E_{B-V}, \mathrm{H}_{2}$ and other hydrides, using optical absorption line observations, and we note the implications of optical studies of the excitation of the ground-state $\mathrm{CH} \Lambda$-doublet for microwave observations. In Sect. 3 we describe our new $9 \mathrm{~cm}$ observations of the $3335 \mathrm{MHz} \mathrm{CH} F=1-1$ (main) line, and compare them to our prior results for $\mathrm{OH}, \mathrm{HCO}^{+}, \mathrm{C}_{2} \mathrm{H}$ and $\mathrm{CO}$ in the same directions. Section 4 is a brief summary.

\section{Optical absorption line studies of $\mathbf{C H}$}

\subsection{The relationship between $\mathrm{CH}$ and $E_{B-V}$}

Much of the original rationale for using $\mathrm{CH}$ as a tracer of $\mathrm{H}_{2}$ was the relationship $N(\mathrm{CH})=6.3 \times 10^{13} \mathrm{~cm}^{-2} E_{B-V}$ derived by Lang \& Willson (1978) using a combination of optical and microwave determinations of $N(\mathrm{CH})$ along some twenty lines of sight; for instance, see Mattila (1986) which forms the basis of the discussion by Magnani \& Onello (1995). The microwave determinations are subject to considerable uncertainty (at least comparatively) so that an optical determination of $N(\mathrm{CH})$ is preferable, at least initially. Lien (1984) shows how to derive $N(\mathrm{CH})$ properly from the available $\mathrm{CH}$ optical absorption lines.

Figure 1 at left shows the run of $N(\mathrm{CH})$ with $E_{B-V}$ found in a much larger sample of more recent optical measurements (Allen 1994; Crane et al. 1995; Crawford 1995; Federman et al. 1994; Gredel et al. 1993; Danks et al. 1984; van Dishoeck \& Black 1989; Crutcher 1985; Jenniskens et al. 1992; Penprase 1993) including the data of Welty et al. tabulated in Rachford et al. (2002). Represented are 140 lines of sight harboring $120 \mathrm{CH}$ detections: many uninformative upper limits contained in the original references were not transcribed. The most recent measurement was used in those cases where lines of sight had been observed more than once. It is not possible to infer a single (or single-valued) or linear relationship between $N(\mathrm{CH})$ and $E_{B-V}$. 


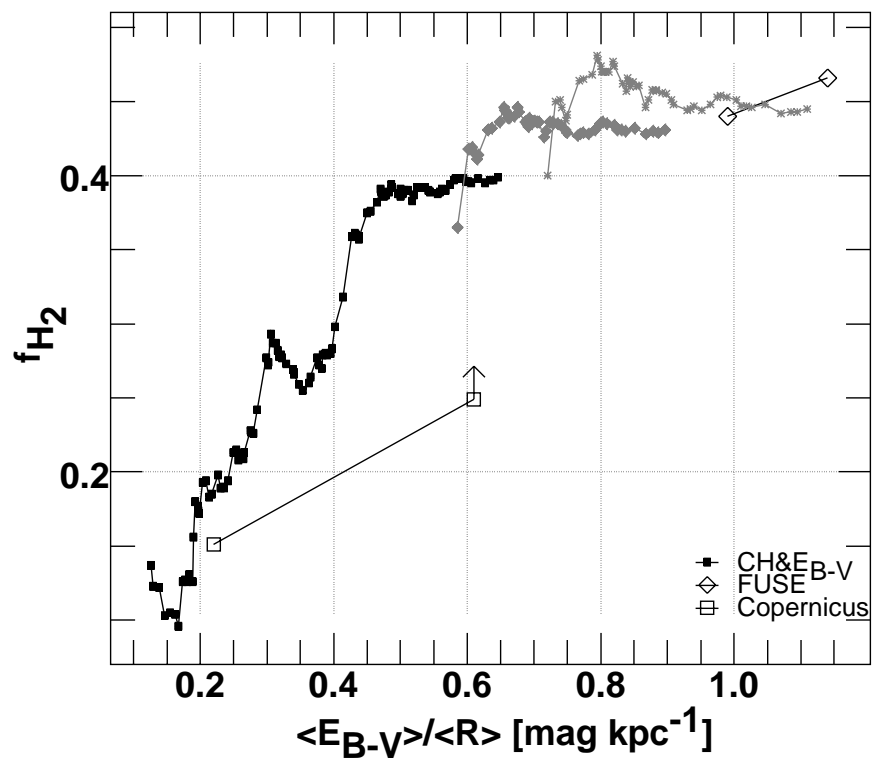

Fig. 2. The fraction of $\mathrm{H}$-nuclei in $\mathrm{H}_{2}$ derived by various means. The connected symbols labeled "Copernicus" represent the results of Bohlin et al. (1978), measured (at left) and corrected for bias to lower than average mean density. The chained curves represent samples of the sightlines with measured $N(\mathrm{CH})$, assuming the same $N(\mathrm{H})-E_{B-V}$ conversion and $X_{\mathrm{CH}}=4.3 \times 10^{-8}$ as described in Sect. 2.2.1

For $E_{B-V}<0.3, N(\mathrm{CH})$ is largely bimodal, i.e. either $N(\mathrm{CH}) \lesssim 3 \times 10^{12} \mathrm{~cm}^{-2}$, in, presumably, typical diffuse gas, or $N(\mathrm{CH})>10^{13} \mathrm{~cm}^{-2}$ in the CO-emission-selected, highlatitude objects studied by Penprase (1993) and along one of the sightlines studied by Crane et al. (1995). In these directions, the gas is apparently dense enough or sufficiently poorlyilluminated for the great majority of hydrogen to have been converted into $\mathrm{H}_{2}$ even for $A_{V}<1$. The transition to consistently high $\mathrm{CH}$ abundances occurs somewhere in the range $0.1 \leq E_{B-V} \leq 0.4$; many lines of sight with $E_{B-V}=0.3 \mathrm{mag}$ do not show $\mathrm{CH}$ and one at $E_{B-V}=0.87$ from Allen (1994) is underabundant compared to the mean by about a factor of 3 .

Many of the points seem to lie along a band increasing approximately as $N(\mathrm{CH}) \propto E_{B-V}^{1.8}$ for $0.1 \leq E_{B-V} \leq 1$; this arises because $N\left(\mathrm{H}_{2}\right)$ increases with $E_{B-V}$ and $X_{\mathrm{CH}} \equiv$ $N(\mathrm{CH}) / N\left(\mathrm{H}_{2}\right)$ is approximately constant (see Sect. 2.2). The enormous variance of the observed $N(\mathrm{CH})$ values near $E_{B-V}=$ $0.3 \mathrm{mag}$ seems quite extraordinary even if order-of-magnitude variations in $N(\mathrm{CH})$ are common at most other $E_{B-V}$ as well. Perhaps a second transition to yet more fully molecular gas will become evident in the $N\left(\mathrm{H}_{2}\right)-E_{B-V}$ relationship as more data become available.

Shown in the left-hand panel of Fig. 1 is the line $N(\mathrm{CH})=$ $3 \times 10^{-8} \times 5.8 \times 10^{21} E_{B-V} \mathrm{~cm}^{-2} / 2$, the maximum $\mathrm{CH}$ column density which could arise if $X_{\mathrm{CH}}=3 \times 10^{-8}$ and all gas along the line of sight were in the form of $\mathrm{H}_{2}$ (i.e. $E_{B-V}$ is converted to a column density of $\mathrm{H}$-nuclei using the standard value of Bohlin et al. 1978). Apparently, a few sightlines with reddening as small as 0.1-0.2 mag have $X_{\mathrm{CH}}$ approaching $10^{-7}$.

Superposed in the left panel of Fig. 1 we have included the mean $\mathrm{CH}-A_{B}$ loci derived for several dark clouds from microwave measurements of the $3335 \mathrm{MHz}$ line, using
$A_{B}=4 E_{B-V}$ as in the original references. In what follows we will denote by $N_{\mu}(\mathrm{CH})$ a $\mathrm{CH}$ column density derived by converting a microwave $\mathrm{CH}$ intensity using standard formulae. For comparison, then, we note that Mattila (1986) used the $3335 \mathrm{MHz}$ line of $\mathrm{CH}$ to derive $N_{\mu}(\mathrm{CH})=(13.9 \pm 1.2) \times$ $10^{13} \mathrm{~cm}^{-2}\left(E_{B-V}-0.085\right)$ and $N_{\mu}(\mathrm{CH})=(13.9 \pm 1.2) \times$ $10^{13} \mathrm{~cm}^{-2}\left(E_{B-V}-0.06\right)$ in the two dark clouds L1590 and L1780. Sandell (1982) found relationships $N_{\mu}(\mathrm{CH})=27.2 \times$ $10^{13} \mathrm{~cm}^{-2}\left(E_{B-V}-0.1\right)$ for L1457 and $N_{\mu}(\mathrm{CH})=9.2 \times$ $10^{13} \mathrm{~cm}^{-2}\left(E_{B-V}-0.03\right)$ for L1172 while the much more limited data for L1642 in Table 4 of Sandell et al. (1981) have a limiting slope of $N_{\mu}(\mathrm{CH}) / E_{B-V}=11.2 \times 10^{13} \mathrm{~cm}^{-2}$.

There is a very substantial range in the limiting behaviour of the derived $N_{\mu}(\mathrm{CH}) / E_{B-V}$ ratios in these objects, and all are much higher than orignally derived in the work of Lang \& Willson (1978). Nonetheless, they do in general correspond fairly well to the values of $X_{\mathrm{CH}}$ seen in the upper reaches of the $\mathrm{CH}$ column densities derived optically. The use of high $N(\mathrm{CH}) / E_{B-V}$ ratios is probably acceptable, even at low $E_{B-V}$, for $\mathrm{CO}$-emitting gas.

\subsection{The relationship between $\mathrm{CH}$ and $\mathrm{H}_{2}$}

Much of the behaviour seen in the left-hand panel of Fig. 1 can be understood simply on the basis of the observed variation of $N\left(\mathrm{H}_{2}\right)$ with reddening, and a fixed or nearly-fixed abundance of $\mathrm{CH}$ relative to $\mathrm{H}_{2}$. Such a nearly linear relationship between $N(\mathrm{CH})$ and $N\left(\mathrm{H}_{2}\right)$ was demonstrated by Federman (1982) and by Danks et al. (1984) and relatively little had changed in the intervening time vis-a-vis $\mathrm{H}_{2}$ until the recent FUSE survey work of Rachford et al. (2002). The CH profiles have improved somewhat in quality and number and the important reanalysis by Lien (1984) clarified the interpretation greatly. Figure 1 at right shows the current situation, from which, for the lines of sight with detections of both $\mathrm{CH}$ and $\mathrm{H}_{2}$, we derive $\log N(\mathrm{CH})=(-7.35 \pm 1.31)+(1.00 \pm 0.06) \log N\left(\mathrm{H}_{2}\right)$. A less comprehensive version of this diagram which provided very nearly the same regression line was shown by Magnani et al. (1998).

In the mean, $X_{\mathrm{CH}}=4.3 \pm 1.9 \times 10^{-8}$. The $\pm 45 \%$ variance of $N(\mathrm{CH})$ about $N\left(\mathrm{H}_{2}\right)$ is appreciable; it sets the ultimate limit on expectations of the reliability of $\mathrm{CH}$ as a predictor of $\mathrm{H}_{2}$. These data represent sightline averages over multiple components and do not prove that the $\mathrm{CH}$ abundance is constant in individual clouds of higher density and extinction. Indeed, the dark cloud data discussed in Sect. 2.4 show that $X_{\mathrm{CH}}$ declines markedly in darker gas and a steady decline from diffuse to dark conditions has been noted since the original microwave surveys of Rydbeck et al. (1976), Hjalmarson et al. (1977) and Mattila (1986). The diminished scatter in Fig. 2 at right for the lines of sight having the highest $N\left(\mathrm{H}_{2}\right)$ is strongly suggestive of blending.

As shown in the inset in the right-hand panel of Fig. 1, much of the scatter in the plot of $N(\mathrm{CH})$ vs. $E_{B-V}$ can be understood simply in terms of the variation of $N\left(\mathrm{H}_{2}\right)$ with $E_{B-V}$, given that $X_{\mathrm{CH}}$ is approximately constant. Along lines of sight where $\mathrm{H}_{2}$ is known directly, $N\left(\mathrm{H}_{2}\right)$ increases approximately as 
Table 1. Column densities of hydrides in diffuse and dark gas.

\begin{tabular}{ccccccc}
\hline \hline & $\zeta$ Oph & $\zeta$ Per & $o$ Per & HD27778 & TMC-1 & ${\mathrm{L} 134 N^{5}}^{5}$ \\
\hline & $N\left(\mathrm{~cm}^{-2}\right)$ & $N\left(\mathrm{~cm}^{-2}\right)$ & $N\left(\mathrm{~cm}^{-2}\right)$ & $N\left(\mathrm{~cm}^{-2}\right)$ & $N_{\mu}\left(\mathrm{cm}^{-2}\right)$ & $N_{\mu}\left(\mathrm{cm}^{-2}\right)$ \\
\hline $\mathrm{CH}^{1}$ & $2.50 \mathrm{E} 13$ & $2.03 \mathrm{E} 13$ & $1.90 \mathrm{E} 13$ & $2.90 \mathrm{E} 13$ & $2 \mathrm{E} 14$ & $1 \mathrm{E} 14$ \\
$\mathrm{NH}^{2}$ & $8.8(1.2) \mathrm{E} 11$ & $9.0(0.2) \mathrm{E} 11$ & & $2.7(0.6) \mathrm{E} 12$ & & \\
$\mathrm{OH}^{3}$ & $4.7(0.7) \mathrm{E} 13$ & $4.1(0.4) \mathrm{E} 13$ & $7.8(2.6) \mathrm{E} 13$ & $10.2(0.4) \mathrm{E} 13$ & $3 \mathrm{E} 15$ & $7.5 \mathrm{E} 14$ \\
$\mathrm{H}_{2}{ }^{3}$ & $4.4 \mathrm{E} 20$ & $4.8 \mathrm{E} 20$ & $4.0 \mathrm{E} 20$ & $1.0(0.3) \mathrm{E} 21$ & $(1 \mathrm{E} 22)$ & $(1 \mathrm{E} 22)$ \\
\hline
\end{tabular}

${ }^{1} N(\mathrm{CH})$ from Crane et al. (1995) except HD27778 from Federman et al. (1994).

${ }^{2} N(\mathrm{NH})$ from Meyer \& Roth (1991) except $\zeta$ Oph from Crawford \& Williams (1997).

${ }^{3} N(\mathrm{OH})$ from Roueff (1996) and Felenbok \& Roueff (1996).

${ }^{4} N\left(\mathrm{H}_{2}\right)$ from Savage et al. (1977) except HD27778 from Joseph et al. (1986).

${ }^{5}$ Dark-cloud data from Ohishi et al. (1992).

$E_{B-V}^{1.8}$ for $E_{B-V}>0.2$. The Penprase (1993) sample, selected on the basis of relatively strong CO $J=1-0$ emission, probably represents a special circumstance whereby the molecular fraction is high even below $E_{B-V}=0.3$.

\subsubsection{The fraction of gas in molecular form}

The fraction of gas in molecular form in the local ISM is an important quantity which provides interesting constraints on interpretations of atomic and molecular gas constituents independently. Here we take a new approach to determining a local mean for $f_{\mathrm{H}_{2}} \equiv 2 N\left(\mathrm{H}_{2}\right) /\left(N(\mathrm{H} \mathrm{I})+2 N\left(\mathrm{H}_{2}\right)\right)$, based on the near-constancy of $X_{\mathrm{CH}}$ and the existence of a substantial set of $\mathrm{CH}$ measurements which sample a wider range of mean lineof-sight densities than has been directly observable in $\mathrm{H} \mathrm{I}$ and $\mathrm{H}_{2}$ : a sample whose mean density is more nearly equal to that of the true mean in the local ISM offers the possibility that the mean molecular gas fraction might be more representative of the overall molecular fraction in the nearby ISM as well.

Shown in Fig. 2 are the results of estimating $\left\langle f_{\mathrm{H}_{2}}\right\rangle$ in three ways. The symbols labelled "Copernicus" are taken from Bohlin et al. (1978) and represent their results along nearly 100 lines of sight of much lower than average mean density $\left\langle E_{B-V}>/<R>=0.22 \mathrm{mag} \mathrm{kpc}^{-1}\right.$. The accepted mean is $0.61 \mathrm{mag} \mathrm{kpc}^{-1}$, from Spitzer (1978) based on the earlier discussion of Münch (1952) ${ }^{1}$. The symbol at lower mean density is the actual measurement; at higher mean density it is their estimate of the true value, corrected for bias. The symbols labelled "FUSE" represent the new data of Rachford et al. (2002); the symbol at lower mean density represents only those lines of sight for which $N(\mathrm{H} \mathrm{I})$ was not estimated by assuming a proportionality to $E_{B-V}$. The chained lines labelled "CH\& $E_{B-V}$ " represent samples of the lines of sight with $\mathrm{CH}$ measurements, assuming $N\left(\mathrm{H}_{2}\right)=N(\mathrm{CH}) / 4.3 \times 10^{-8}$ and $N(\mathrm{H})=N(\mathrm{H} \mathrm{I})+2 N\left(\mathrm{H}_{2}\right)=5.8 \times 10^{21} \mathrm{~cm}^{-2} E_{B-V}$, so that $<N\left(\mathrm{H}_{2}\right)>/<N(\mathrm{H})>=4.01 \times 10^{-15}<N(\mathrm{CH})>/<E_{B-V}>$, as we now discuss.

To construct the $\mathrm{CH}$-based samples, we sorted the lines of sight in order of increasing individual $E_{B-V} / R$ and derived

\footnotetext{
${ }^{1}$ Reddening per unit distance has units of density if $E_{B-V} \propto N(\mathrm{H})$; $R$ is the distance to a background star. See Spitzer (1978).
}

$<N\left(\mathrm{H}_{2}\right)>/<N(\mathrm{H})>$ for contiguous, progressively larger samples of four or more sightlines beginning at different lower cutoffs. Where only upper limits on $N(\mathrm{CH})$ were available, $N\left(\mathrm{H}_{2}\right)$ was taken to be zero. So each chained line segment in Fig. 2 shows the mean molecular fraction, derived under the idealized assumption of a constant $\mathrm{CH}$ abundance and gas-dust ratio, as the sample mean extinction per unit distance varies. The dark, left-most line labelled " $\mathrm{CH} \& E_{B-V}$ " begins at the lowest observed $E_{B-V} / R$ and contains all lines of sight at its rightmost extent; it just barely extends beyond $\left\langle E_{B-V}\right\rangle \mid\langle R\rangle=$ $0.61 \mathrm{mag} \mathrm{kpc}^{-1}$ when all the lines of sight are included.

The Copernicus and FUSE measurements (not the "corrected" Copernicus estimate) agree entirely with the $\mathrm{CH}$-based samples; the high mean molecular fraction seen by FUSE should not be dismissed as biased because of the high sample mean density. From the $\mathrm{CH}$ data we see that the mean molecular fraction appears to increase fairly rapidly at low sample mean densities and is of order $0.4-0.45$ at the accepted local mean density.

\subsection{Population ratios}

As noted in the Introduction, the excitation temperatures of the microwave $\mathrm{CH}$ transitions, needed to convert observed brightnesses to column density in diffuse gas, have not been (and probably cannot be) determined from microwave observations. However, Lien (1984) pointed out that the populations of the upper and lower levels of the ground-state $\mathrm{CH} \Lambda$-doublet could be derived separately by observing different optical absorption lines (see his Fig. 2). He derived the excitation temperature $T_{\text {exc }}$ from extant observations toward several well-studied stars and found that it could be either slightly negative or positive, but with rather large uncertainty. Extant models of $\mathrm{CH}$ excitation by Bertojo et al. (1976) predicted a transition from normal excitation $\left(T_{\mathrm{exc}}>0 \mathrm{~K}\right)$ to substantial inversion $\left(T_{\mathrm{exc}}=-0.6 \mathrm{~K}\right)$ at a density somwehere between $10^{2}$ and $10^{3} \mathrm{H}_{2} \mathrm{~cm}^{-3}$, so it was (and is) reasonable to believe that both branches of the excitation conditions would manifest themselves in diffuse gas.

Jura \& Meyer (1985) rose to the challenge of taking data sufficiently accurate for this purpose and their results are summarized in Fig. 3 here. The horizontal axis is the excitation temperature of the $\Lambda$-doublet (the optical structure does not 


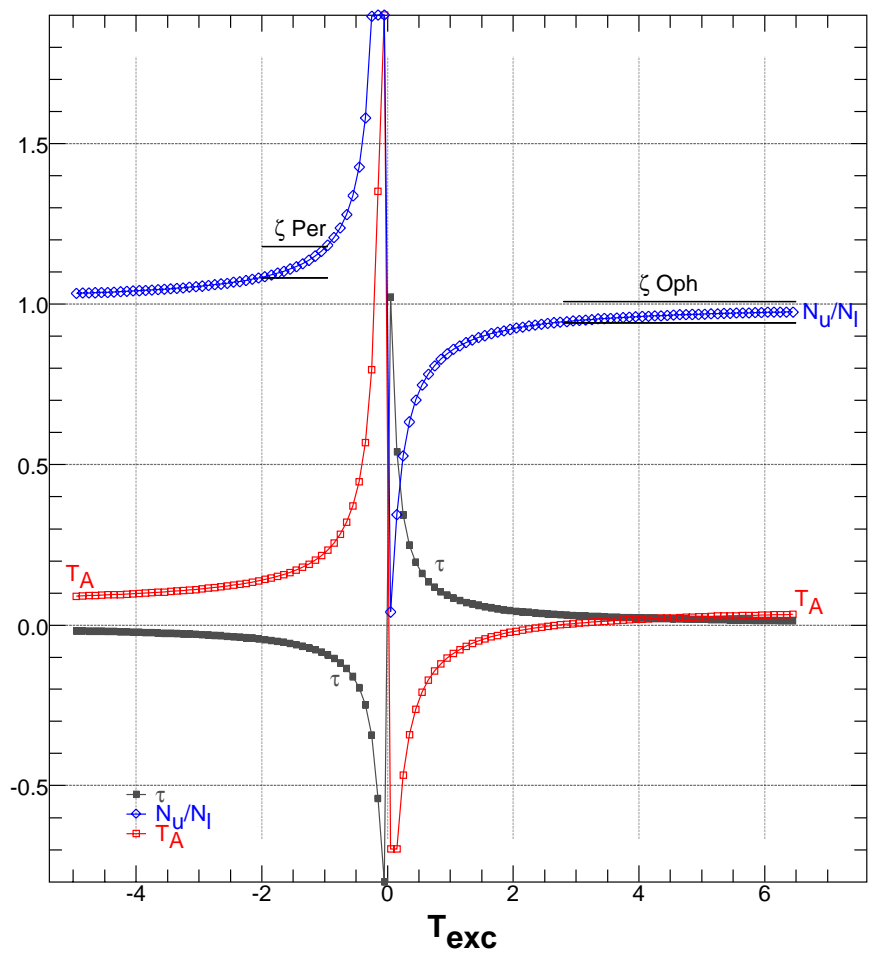

Fig. 3. Population of the $\mathrm{CH} \Lambda$-doublet. Plotted as a function of the excitation temperature of the $\mathrm{CH}$ ground-state $\Lambda$-doublet are the population ratio of the upper and lower states $N_{\mathrm{u}} / N_{\mathrm{l}}$ (upper curve; chained diamonds) and the optical depth (shaded) and antenna temperature of the $3335 \mathrm{MHz} F=1-1$ transition for $N(\mathrm{CH}) / \Delta V=\left(N_{\mathrm{u}}+N_{\mathrm{l}}\right) / \Delta V=$ $2.5 \times 10^{13} \mathrm{~cm}^{-2}\left(\mathrm{~km} \mathrm{~s}^{-1}\right)^{-1}, \eta_{B}=0.7$. The horizontal bars labelled " $\zeta$ Per" and " $\zeta$ Oph" represent the $\pm 1 \sigma$ range of population ratios observed by Jura \& Meyer (1985). See Sect. 2.3 of the text.

resolve the hyperfine splitting). We define $T_{\text {exc }}$ in the usual way by asserting that the upper and lower halves of the doublet (which have equal statistical weights) are in the ratio $N_{\mathrm{u}} / N_{\mathrm{l}}=\exp \left(-h v / k T_{\text {exc }}\right)$ with $v=3335 \mathrm{MHz}, h v / k=0.160 \mathrm{~K}$ corresponding to the so-called main $(F=1-1)$ line. We further assume that the excitation temperature of the doublet as a whole applies to the microwave lines individually, and plot the antenna temperature and optical depth of the $F=1-1$ line assuming a beam efficiency of $\eta_{B}=0.7$ and $N(\mathrm{CH}) / \Delta V=$ $\left(N_{\mathrm{u}}+N_{\mathrm{l}}\right) / \Delta V=2.5 \times 10^{13} \mathrm{~cm}^{-2}\left(\mathrm{~km} \mathrm{~s}^{-1}\right)^{-1}$, using the column density observed toward $\zeta$ Oph (see Table 1; formulae sufficient to reproduce this plot are given in Sect. 3.1). The plotted curves then represent the hypothetical antenna temperature and optical depth of a $1 \mathrm{~km} \mathrm{~s}^{-1}$-wide line, or the profile integral of either quantity if $|\tau|$ is small.

Around the curves of $N_{\mathrm{u}} / N_{\mathrm{l}}$ we have placed horizontal error bars displaced by $\pm 1 \sigma$ about the mean for the lines of sight toward $\zeta$ Per and $\zeta$ Oph. Jura \& Meyer (1985) found two components with similar conditions toward $\zeta$ Per; their results for $o$ Per (not represented here) are similar to those for $\zeta$ Oph. For $\zeta$ Per, the error bars extend to meet the curve of population ratio and are meant to show the $\pm 1 \sigma$ error in $T_{\text {exc }}$. For $\zeta$ Oph the case cannot be summarized quite so concisely. The $-1 \sigma$ error bar actually extends indefinitely to the right since the population ratio is bounded above at unity for $T_{\mathrm{exc}}>0$.
The $+1 \sigma$ error bar for $\zeta$ Oph also has a branch to the far left (since it lies above unity in the population ratio) and population ratios above unity are consistent with the $\zeta$ Oph measurements at about the $1 \sigma$ level.

The optically-derived population ratios nominally predict that the microwave brightness should be much larger for $\zeta$ Per; a factor three is cited by Jura \& Meyer (1985), even given that $N(\mathrm{CH})$ is $25 \%$ higher toward $\zeta$ Oph. However, this is not observed to be the case. Both directions have main-line profile integrals (integrated antenna temperature) of $0.08-0.09 \mathrm{~K} \mathrm{~km} \mathrm{~s}^{-1}$ if our results for $\zeta$ Oph (shown below and in Liszt 1997) can be compared to those of Hjalmarson et al. (1977) or Willson (1981) for $\zeta$ Per. Unfortunately, this similarity was obscured for Jura \& Meyer (1985) because Lien (1984) quoted a profile integral which was twice as large for $\zeta$ Per as for $\zeta$ Oph.

It appears that the $3335 \mathrm{MHz} \mathrm{CH}$ line toward both stars is inverted, with (nominally) $T_{\mathrm{exc}} \approx-5 \mathrm{~K}$ for $\zeta$ Oph and $T_{\mathrm{exc}} \approx$ $-3 \mathrm{~K}$ for $\zeta$ Per, if the broad microwave and narrow optical lines of sight sample approximately equal values of $N(\mathrm{CH})^{2}$. These $\left|T_{\text {exc }}\right|$ are small enough that a noticeable error occurs if $N_{\mu}(\mathrm{CH})$ is derived in the limit $T_{\text {exc }} \rightarrow-\infty ; N(\mathrm{CH})$ would be overestimated by $60-100 \%$.

In summary, even though the radio data do not show the most extreme differences in excitation suggested (but not absolutely required) by the optical results, $\mathrm{CH}$ in the diffuse ISM could in principle be inverted (perhaps fairly strongly) or not, and we should be prepared to encounter situations where the the same $N(\mathrm{CH})$ can produce different microwave $\mathrm{CH}$ profile integrals and $N_{\mu}(\mathrm{CH})$. The usual practice of deriving $N_{\mu}(\mathrm{CH})$ in the limit of weak inversion is not necessarily appropriate in all diffuse gas.

\subsection{Abundances of hydrides in diffuse and dark gas}

Shown in Table 1 are the optically-determined column densities of the simple hydrides of $\mathrm{C}, \mathrm{N}$, and $\mathrm{O}$ in the only directions in which they may be compared. Taking weighted averages, we have for the means and their formal variance - taken over a very limited range of $N\left(\mathrm{H}_{2}\right)-N(\mathrm{CH}) / N\left(\mathrm{H}_{2}\right), 4.4 \pm 1.0 \times 10^{-8}$; $N(\mathrm{OH}) / N\left(\mathrm{H}_{2}\right), 1.0 \pm 0.2 \times 10^{-7} ; N(\mathrm{NH}) / N\left(\mathrm{H}_{2}\right), 1.9 \pm 0.1 \times 10^{-9}$; $N(\mathrm{OH}) / N(\mathrm{CH}), 3.0 \pm 0.9 ; N(\mathrm{OH}) / N(\mathrm{NH}), 50.9 \pm 5.1$.

Also shown are the microwave-determined column densities of $\mathrm{OH}$ and $\mathrm{CH}$ toward TMC-1 and L134N, taken from Ohishi et al. (1992). $X_{\mathrm{CH}}$ declines markedly (a factor of 2-4) when carbon is in the form of $\mathrm{CO}$ rather than $\mathrm{C}^{+}$, while $X_{\mathrm{OH}}$ remains constant or increases slightly.

\section{Microwave $\mathrm{CH}$ lines in diffuse gas}

\subsection{Data and new $3335 \mathrm{MHz}$ observations}

The new $3335 \mathrm{MHz} F=1-1 \mathrm{CH}$ observations discussed here were taken with the now-defunct NRAO 43 m antenna in 1995 September. The data taken toward $\zeta$ Oph were reported previously in Liszt (1997). The continuum background sources

\footnotetext{
2 That is, we may infer comparable excitation from the radio data only if $N(\mathrm{CH})$ is similar in the two directions, as is the case along the narrow optical lines of sight; see also Willson (1981).
} 

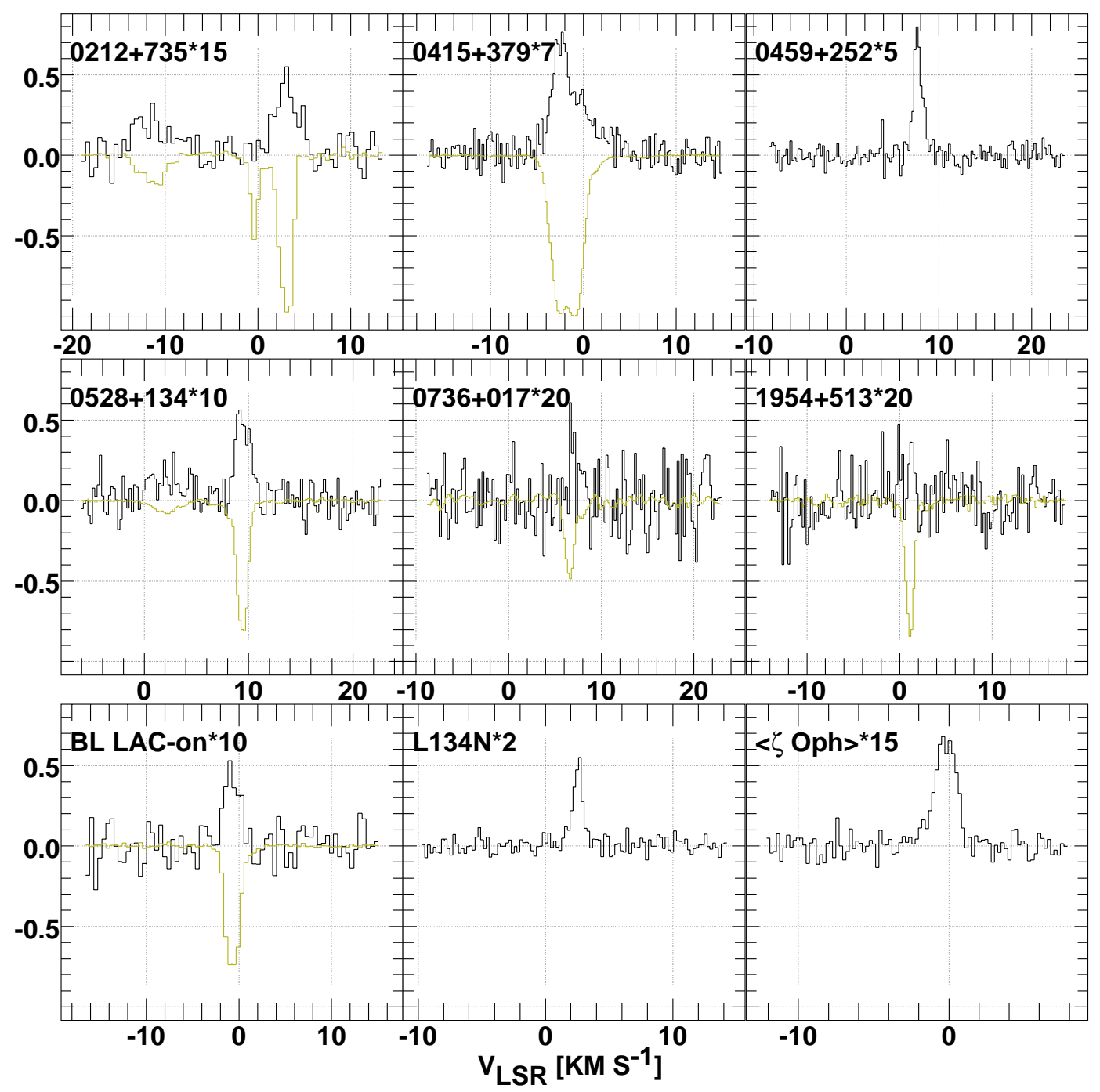

Fig. 4. $\mathrm{CH}$ emission profiles (units of Kelvins). For the lines of sight around continuum sources, the profiles are averages of off-source spectra taken 11' North, East, South and West (except for BL Lac, which is on-source). Shown shaded, where possible, is the corresponding $89 \mathrm{GHz}$ $\mathrm{HCO}^{+} J=1-0$ absorption profile from Lucas \& Liszt (1996) in the form line/continuum-1. At lower right is a spectrum averaged over 80' in a North-South direction around $\zeta$ Oph from Liszt (1997), and a comparison spectrum toward L134N. The emission spectra have been scaled in various ways, as indicated.

Table 2. Background sources observed.

\begin{tabular}{lccc}
\hline \hline Source & $l$ & $b$ & $T_{\mathrm{C}}{ }^{1}$ \\
\hline $\mathrm{B} 0212+735$ & $128.93^{\circ}$ & $11.96^{\circ}$ & $0.79 \mathrm{~K}$ \\
$\mathrm{~B} 0224+671$ & $132.12^{\circ}$ & $6.23^{\circ}$ & $0.56 \mathrm{~K}$ \\
$\mathrm{~B} 0355+508$ & $150.38^{\circ}$ & $-1.60^{\circ}$ & $0.90 \mathrm{~K}$ \\
$\mathrm{~B} 0415+379$ & $161.68^{\circ}$ & $-8.82^{\circ}$ & $2.79 \mathrm{~K}$ \\
$\mathrm{~B} 0433+295$ & $170.58^{\circ}$ & $-11.66^{\circ}$ & $7.87 \mathrm{~K}$ \\
$\mathrm{~B} 0459+252$ & $177.73^{\circ}$ & $-9.91^{\circ}$ & $1.02 \mathrm{~K}$ \\
$\mathrm{~B} 0528+134$ & $191.37^{\circ}$ & $-11.01^{\circ}$ & $0.75 \mathrm{~K}$ \\
$\mathrm{~B} 0727-115$ & $227.77^{\circ}$ & $3.14^{\circ}$ & $0.91 \mathrm{~K}$ \\
$\mathrm{~B} 0736+017$ & $216.99^{\circ}$ & $11.38^{\circ}$ & $0.68 \mathrm{~K}$ \\
$\mathrm{~B} 0954+658$ & $145.76^{\circ}$ & $43.13^{\circ}$ & \\
$\mathrm{B} 1954+513$ & $85.20^{\circ}$ & $11.90^{\circ}$ & $0.44 \mathrm{~K}$ \\
$\mathrm{~B} 2013+370$ & $74.77^{\circ}$ & $1.36^{\circ}$ & $1.95 \mathrm{~K}$ \\
$\mathrm{~B} 2023+336$ & $73.03^{\circ}$ & $-2.23^{\circ}$ & $0.85 \mathrm{~K}$ \\
B2200+420 & $92.13^{\circ}$ & $-10.40^{\circ}$ & $1.69 \mathrm{~K}$ \\
\hline
\end{tabular}

${ }^{1}$ Gain was approximately $2.5 \mathrm{Jy} \mathrm{K}^{-1}$. observed are given in Table 2; with the exceptions of $3 \mathrm{C} 123$ (B0433+295) and 3C133 (B0459+252) they are all strong mmwave sources. The observing strategy was like that followed for OH (Liszt \& Lucas 1996) and $\mathrm{H}_{2} \mathrm{CO}$ (Liszt \& Lucas 1995) whereby we observed toward the source and 1.2 HPBW (here, $1.2 \times 9^{\prime}$ ) displaced in the four cardinal directions. The beam efficiency of the telescope $\eta_{B}$ is usually quoted as $0.7-0.8$. We take $\eta_{B}=0.7$ because on this scale our profile toward L134N reproduces the canonical value for $N_{\mu}(\mathrm{CH})$ cited in Table 1 . We used a channel separation of $2.44 \mathrm{kHz}$ or $0.22 \mathrm{~km} \mathrm{~s}^{-1}$. The off-source system temperature was typically $38-42 \mathrm{~K}$.

The relationship between brightness of the microwave lines and $\mathrm{CH}$ column is nearly always taken in the Rayleigh-Jeans limit for small optical depth because $3335 \mathrm{MHz}$ corresponds to $0.160 \mathrm{~K}$ and the excitation temperature is usually taken as either $-15 \mathrm{~K}$, following the original observation of Perseus and Orion Arm features toward Cas A (Rydbeck et al. 1976; Hjalmarson et al. 1977) or $-60 \mathrm{~K}$ (Genzel et al. 1979). These 

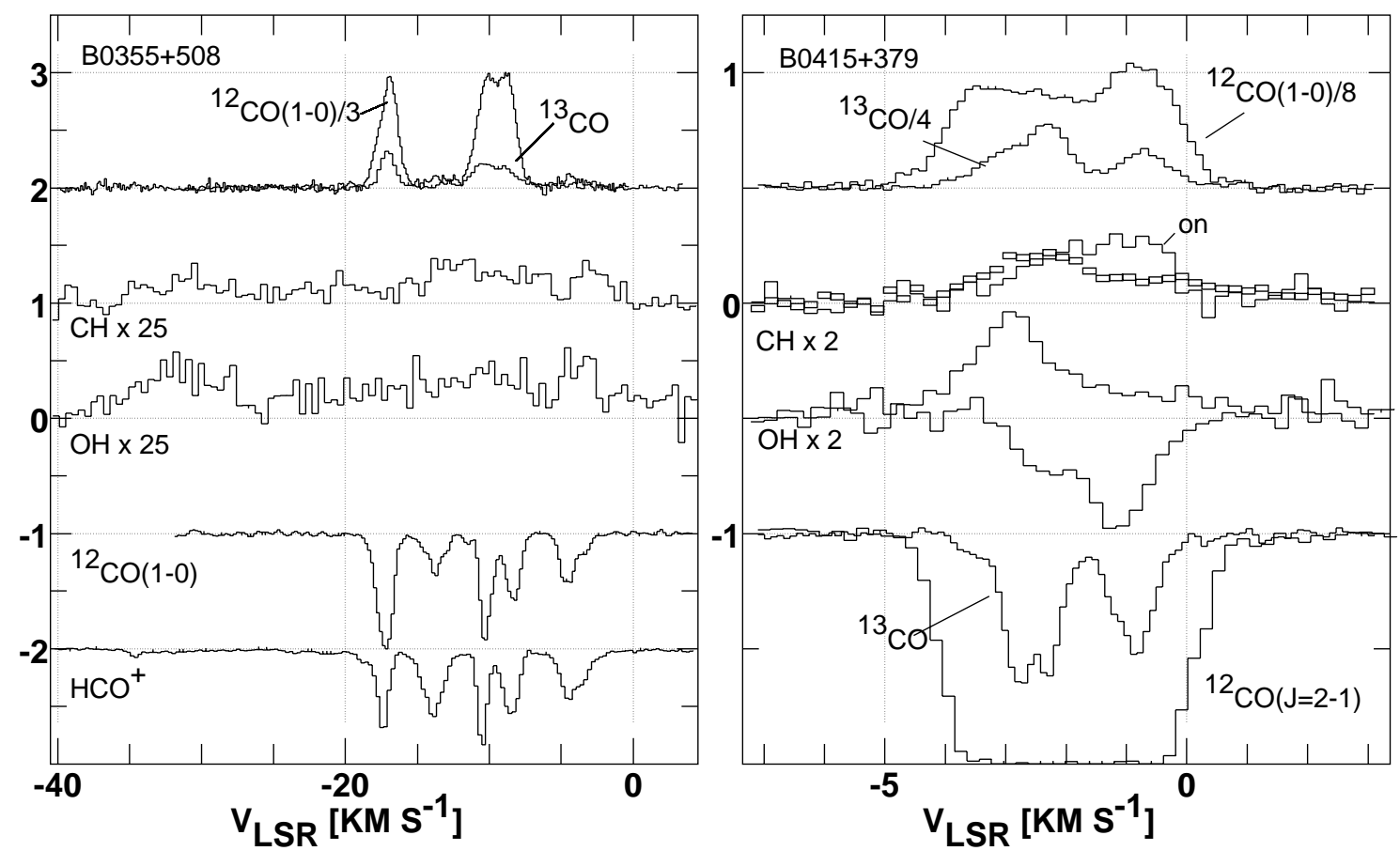

Fig. 5. Spectra of $\mathrm{CH}$ and other species toward B0355+508 (left) and B0415+379. For B0415+379 (3C111) both on-source and off-source spectra are shown for $\mathrm{CH}$ and $\mathrm{OH}$. The on-source $\mathrm{OH}$ spectrum and all the other obvious absorption spectrum are presented in the form (line/continuum-1) while the on-source $\mathrm{CH}$ spectra and all the obvious emission spectra are in $\mathrm{K}$. At right, the $\mathrm{CO}$ emission spectra at top are for $J=1-0$ while the absorption spectra at bottom are $J=2-1$.

values imply that the optical depth is very low, because typical brightnesses are $0.05 \mathrm{~K}$. From atomic physics and thermodynamic equilbrium we have for the $v=3335 \mathrm{MHz} F^{\prime}=1 \rightarrow$ $F^{\prime \prime}=1$ transition the relationship

$\int \tau_{11} \mathrm{~d} v=\frac{0.75 \mathrm{~N}_{l} \lambda^{3} A_{11}\left(1-\exp \left(-h v / k T_{\text {exc }}\right)\right)}{8 \pi}$

where $\lambda=c / v, A_{11}=2.04 \times 10^{-10} \mathrm{~s}^{-1}$ and $N_{1}=N(\mathrm{CH}) /(1+$ $\left.\exp \left(-h v / k T_{\text {exc }}\right)\right)$ is the population in the lower half of the ground-state $\Lambda$-doublet, of which a fraction $3 / 4$ is in the $F^{\prime \prime}=$ 1 level.

This may be used in concert with the relationship between the observed microwave antenna temperature $T_{\mathrm{A}}$, the excitation temperature $T_{\text {exc }}$ and the background continuum temperature against which the line is observed $T_{\mathrm{bg}}$

$T_{\mathrm{A}}=\eta_{B}\left(1-\mathrm{e}^{-\tau}\right)\left[J\left(T_{\mathrm{exc}}\right)-J\left(T_{\mathrm{bg}}\right)\right]$

where $J(x) \equiv(h v / k) /(\exp (h v / k x)-1)$. In the RayleighJeans limit of small optical depth, with $N(\mathrm{CH}) /(1+$ $\left.\exp \left(-h v / k T_{\text {exc }}\right)\right) \approx N(\mathrm{CH}) / 2$ one arrives at the relationship

$N_{\mu}(\mathrm{CH})=\frac{2.82 \times 10^{14} \mathrm{~cm}^{-2} T_{\text {exc }} \int T_{\mathrm{A}} \mathrm{d} v}{\eta_{B}\left(T_{\text {exc }}-T_{\mathrm{bg}}\right)}$.

In the literature, the mantissa of the constant on the right hand side of this expression is sometimes quoted as 2.9 , rather than 2.82 .

Mean off-source spectra for many lines of sight are shown in Fig. 4 along with $\mathrm{HCO}^{+}$absorption data (Lucas \& Liszt 1996) where possible. Except in two cases (see Sect. 3.4) the on and off-source $\mathrm{CH}$ spectra do not differ significantly and the latter have lower noise levels since they are fourpoint averages. For BL Lac (B2200+420) we detected CH only toward the source as was also the case in our search for $\mathrm{HCO}^{+}$emission. Also shown are a comparison spectrum toward $\mathrm{L} 134 \mathrm{~N}$ as well as the mean of the nine spectra observed around $\zeta$ Oph and shown individually in Liszt (1997). Line profile integrals and fitted gaussian components are given in the Appendix. $\mathrm{CH}$ spectra toward two sources are compared with $\mathrm{OH}, \mathrm{HCO}^{+}$and $\mathrm{CO}$ emission and absorption in Fig. 5.

Figure 4 shows that most but not all $\mathrm{HCO}^{+}$absorption components are seen in $\mathrm{CH}$ emission: the $\mathrm{HCO}^{+}$absorption feature toward $\mathrm{B} 0212+735$ which is missing in $\mathrm{CH}$ emission is also absent in CO emission, but seen in CO absorption (Liszt \& Lucas 1998). The absence of $\mathrm{CH}$ absorption hinders our ability to find $N_{\mu}(\mathrm{CH})$ for components of low column density. Given the vast difference in resolution, the differences in $\mathrm{CH}$ and $\mathrm{HCO}^{+}$profile shapes however interesting, are remarkably slight.

Figure 5 toward $\mathrm{B} 0355+508$ shows that $\mathrm{CH}$, like $\mathrm{OH}$, has a diffuse distributed component and does not clearly distinguish individual features in all cases. As discussed by Liszt \& Lucas (2000), $\mathrm{HCO}^{+}$absorption extends out to $-36 \mathrm{~km} \mathrm{~s}^{-1}$, corresponding to the main body of $\mathrm{H} \mathrm{I}$ absorption in this direction.

Toward 3C111 (B0415+379) the $\mathrm{CH}$ is strongly inverted only in the component at higher velocity, which is more chemically complex and likely more nearly fully molecular (see the discussion in Lucas \& Liszt 1998). Gas in the lower-velocity component is heavily fractionated in carbon, indicating that only a small fraction of the carbon is in CO. Note that the $\mathrm{CO}$ absorption profiles at bottom are for the $\mathrm{CO} J=2-1$ line while the $\mathrm{CO}$ emission data shown at top are for $J=1-0$. 

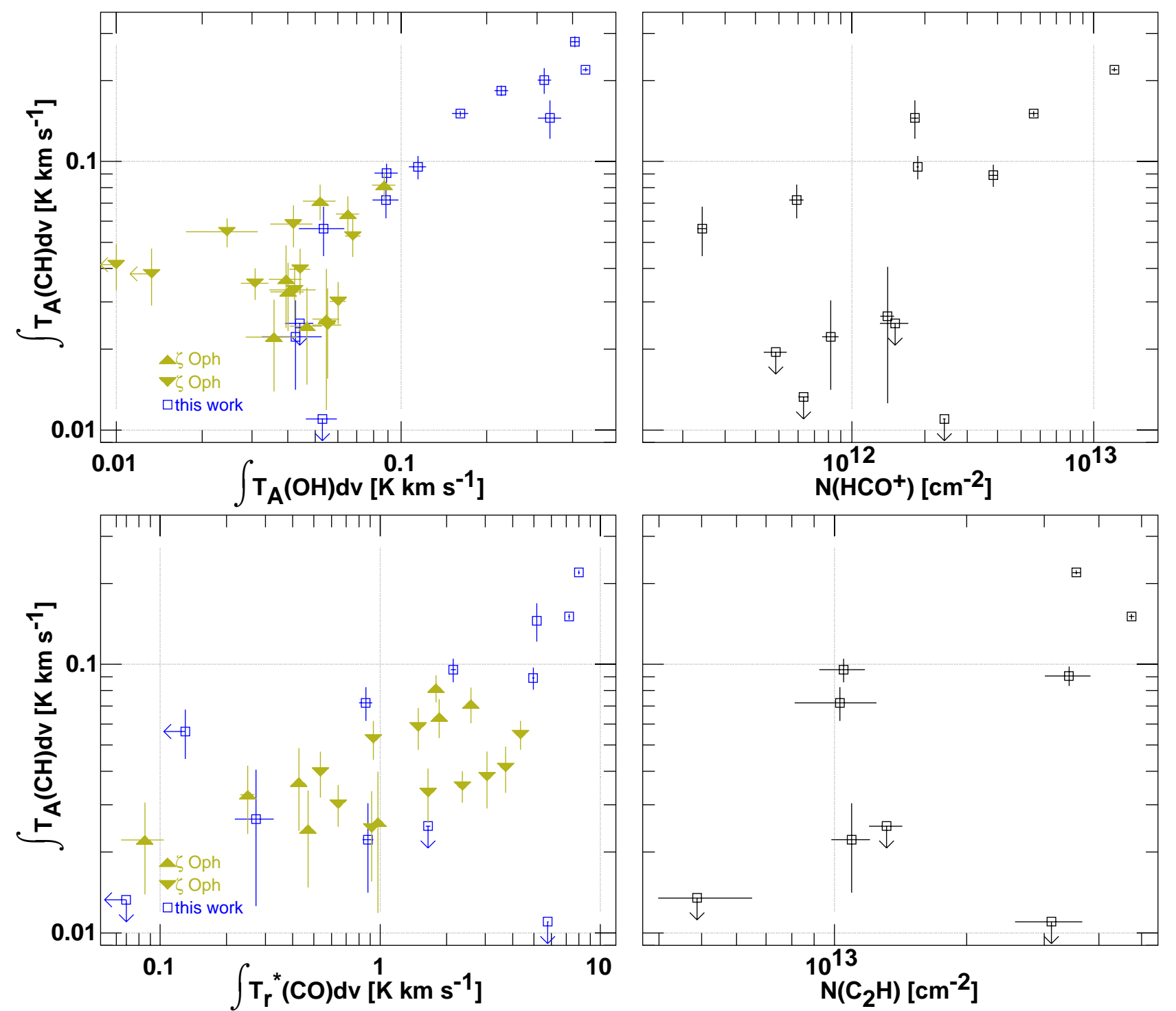

Fig. 6. Comparison of $3335 \mathrm{MHz} F=1-1 \mathrm{CH}$ profile integrals with other quantities. Left top: comparison with the OH profile integral toward compact continuum sources (Lucas \& Liszt 1996; Liszt \& Lucas 1996, 2000) and (heavily shaded) the data of Liszt (1997) around $\zeta$ Oph. Left bottom: the CO $J=1-0$ profile from our 12m telescope data (Liszt \& Lucas 1998) and (grayed) the CO data around $\zeta$ Oph from Liszt (1997) (see Sect. 2.3). Right top: the $\mathrm{HCO}^{+}$column density derived from $89 \mathrm{GHz}$ absorption (Lucas \& Liszt 1996). Right bottom: the $\mathrm{C}_{2} \mathrm{H}$ column density from mm-wave absorption (Lucas \& Liszt 2000a).

For a discussion of $\mathrm{CO}$ along this line of sight, see Lucas \& Liszt (1998) and Liszt \& Lucas (1998).

\section{2. $\mathrm{CH}, \mathrm{OH}, \mathrm{HCO}^{+}$and $\mathrm{C}_{2} \mathrm{H}$}

Figure 6 compares the integrated (off-source) $\mathrm{CH}$ line strength with several other measures; the quantities used in the figure are given in Table A. 1 in the Appendix. The $\mathrm{HCO}^{+}$and $\mathrm{C}_{2} \mathrm{H}$ absorption (Lucas \& Liszt 1996, 2000a) and CO emission (Liszt \& Lucas 1998), were taken on-source while the $\mathrm{OH}$ emission for continuum sources (Liszt \& Lucas 1996, 2000) is also offsource data, but displaced further owing to the lower resolution. In this figure we have also included (heavily greyed) our $\mathrm{CH}, \mathrm{OH}$ and $\mathrm{CO}$ data for the diffuse gas around $\zeta \mathrm{Oph}$ (Liszt 1997). Shown as shaded upward (downward) triangles are the values observed at or to the North (South) of the star; the profile integrals for positive and negative velocity gas are shown separately because there are actually two components at each position. Around $\zeta \mathrm{Oph}$, the observations of $\mathrm{CH}, \mathrm{OH}$ and $\mathrm{CO}$ were concentric. For purposes of nominal comparison $N_{\mu}(\mathrm{CH}) \approx 4 \times 10^{14} \mathrm{~cm}^{-2} \int T_{\mathrm{A}} \mathrm{d} v$ corresponding to $T_{\mathrm{exc}} \ll 0$, $\eta_{B}=0.7$.

The $\mathrm{OH}$ and $\mathrm{CH}$ emission profile integrals are tightly correlated for the stronger components. For these features the mean signal-noise weighted ratio of the $\mathrm{CH}$ and $\mathrm{OH}$ line profile integrals (in the sense $\mathrm{CH}: \mathrm{OH}$ ) is $0.67 \pm 0.19$. Deriving $N_{\mu}(\mathrm{CH})$ as given just above and taking the usual formulae for $N_{\mu}(\mathrm{OH})$ (e.g. Eq. (3) of Liszt \& Lucas 1996) with $T_{\mathrm{exc}}-T_{\mathrm{cmb}}=1$ or $2 \mathrm{~K}$ for $\mathrm{OH}($ ibid $)$, we have that $N_{\mu}(\mathrm{OH}) / N_{\mu}(\mathrm{CH})=4.3$ or 2.8 , in agreement with the result derived optically and quoted in Sect. 2.4, $N(\mathrm{OH}) / N(\mathrm{CH})=3 \pm 0.9$. This serves as a helpful confirmation of the unexpectedly rather small $\mathrm{OH}$ main-line excitation 
temperatures seen in diffuse gas in the radio and optical (Roueff 1996) regimes. Indirectly, it also suggests that the $\mathrm{CH}$ is only weakly inverted $\left(\left|T_{\text {exc }}\right|\right.$ is large).

There appear to be two branches of the $\mathrm{CH} / \mathrm{OH}$ ratio for weak features, one in which the $\mathrm{CH}$ brightness increases fairly abruptly at $\int T_{\mathrm{A}}(\mathrm{OH}) \mathrm{d} v=0.04-0.05 \mathrm{~K} \mathrm{~km} \mathrm{~s}^{-1}$ and another (seen South of $\zeta \mathrm{Oph}$ ) where the $\mathrm{CH}$ is relatively strong at small values of the $\mathrm{OH}$ integral. The weak $\mathrm{CH}$ features showing complicated behaviour in $\mathrm{OH}$ and $\mathrm{CH}$ are double-valued but well-ordered with respect to a comparison of $\mathrm{CH}$ and $\mathrm{CO}$ as discussed in Sect. 3.3.

The comparison with both $\mathrm{HCO}^{+}$and $\mathrm{C}_{2} \mathrm{H}$ absorption seems to show some sort of bimodality or, at least, a very large range of $\mathrm{CH}$ profile integrals at a given $N\left(\mathrm{HCO}^{+}\right)$or $N\left(\mathrm{C}_{2} \mathrm{H}\right)$. If the fractional abundances of $\mathrm{CH}$ and $\mathrm{HCO}^{+}$are both about constant (which is otherwise believed to be the case for diffuse gas) the $3335 \mathrm{MHz}$ profile integral $\left(N_{\mu}(\mathrm{CH})\right)$ does not have a single proportionality to $N(\mathrm{CH})$ for $N\left(\mathrm{HCO}^{+}\right)<3 \times 10^{12} \mathrm{~cm}^{-2}$. Taking the data at face value, for $N\left(\mathrm{HCO}^{+}\right)=10^{12} \mathrm{~cm}^{-2}$ we have that $N_{\mu}(\mathrm{CH}) \approx 0.8-3 \times 10^{13} \mathrm{~cm}^{-2}$ or $N\left(\mathrm{H}_{2}\right)=$ $2-8 \times 10^{20} \mathrm{~cm}^{-2}$, leading to $N\left(\mathrm{HCO}^{+}\right) / N\left(\mathrm{H}_{2}\right)=1.25-5 \times 10^{-9}$. The same comparison at $N\left(\mathrm{HCO}^{+}\right)=3 \times 10^{12} \mathrm{~cm}^{-2}$ and $N_{\mu}(\mathrm{CH}) \approx 5 \times 10^{13} \mathrm{~cm}^{-2}$ yields $N\left(\mathrm{HCO}^{+}\right) / N\left(\mathrm{H}_{2}\right)=2.5 \times 10^{-9}$. Earlier, we inferred $N\left(\mathrm{HCO}^{+}\right) / N\left(\mathrm{H}_{2}\right)=2-3 \times 10^{-9}$ from the observed tight relationship between $\mathrm{HCO}^{+}$and $\mathrm{OH}$ (Lucas \& Liszt 1996; Liszt \& Lucas 1996) and we showed that such an unexpectedly high $\mathrm{HCO}^{+}$abundances suffices to explain the observed quantities of $\mathrm{CO}$ for all $N(\mathrm{CO}) \lessgtr 3 \times 10^{16} \mathrm{~cm}^{-2}$ (Liszt $\&$ Lucas 2000). A cross-comparison of the $N(\mathrm{CN}) / N\left(\mathrm{H}_{2}\right)$ ratios determined optically with the column densities of $\mathrm{CN}$ and $\mathrm{HCO}^{+}$seen at mm-wavelengths by Liszt \& Lucas (2001) also confirmed this abundance of $\mathrm{HCO}^{+}$.

\section{3. $\mathrm{CH}$ and $\mathrm{CO}$}

For the comparison with $\mathrm{CO}$ emission at lower left in Fig. 6, the new data toward continuum sources seemed also to indicate bimodality for the weaker emission. The combined dataset including the points taken around $\zeta$ Oph clearly confirms this behaviour for intermediate values of the $\mathrm{CO}$ emission profile integral $0.3 \leq W(\mathrm{CO}) \leq 5 \mathrm{~K} \mathrm{~km} \mathrm{~s}^{-1}$. The $\mathrm{CO}$ emission profile integral is definitely not bimodal with respect to either $N\left(\mathrm{HCO}^{+}\right)$or $N(\mathrm{CO})$ over this range (Liszt \& Lucas 1998). A direct comparison between the CO column density measured in absorption and the $\mathrm{mm}$-wave emission brightness shows that $N(\mathrm{CO}) \approx 1.25 \times 10^{15} \mathrm{~cm}^{-2} \int T_{\mathrm{r}}^{*} \mathrm{~d} v$ for $\int T_{\mathrm{r}}^{*} \mathrm{~d} v=$ $0.3-4 \mathrm{~K} \mathrm{~km} \mathrm{~s}^{-1}$, see Fig. 12 of Liszt \& Lucas (1998).

When the $\mathrm{CH}$ brightness is assumed to be proportional to the $\mathrm{CH}$ column density, the correlation between $\mathrm{CH}$ and $\mathrm{H}_{2}$ then provides for a $\mathrm{CO}$ brightness $-\mathrm{H}_{2}$ column density conversion as well. Magnani \& Onello (1995) recently compared brightnesses of $\mathrm{CH}$ and $\mathrm{CO}$, deriving $N\left(\mathrm{H}_{2}\right)$ from $N_{\mu}(\mathrm{CH})$ by use of the $\mathrm{CH}-\mathrm{H}_{2}$ conversion of Mattila (1986) as shown in Fig. 1 and discussed in Sect. 2.1 here. Magnani \& Onello (1995) noted a lot of scatter in the CO brightness $-\mathrm{H}_{2}$ column density conversion factors so derived, but did not plot the underlying data, which appear in graphical form here in Fig. 7

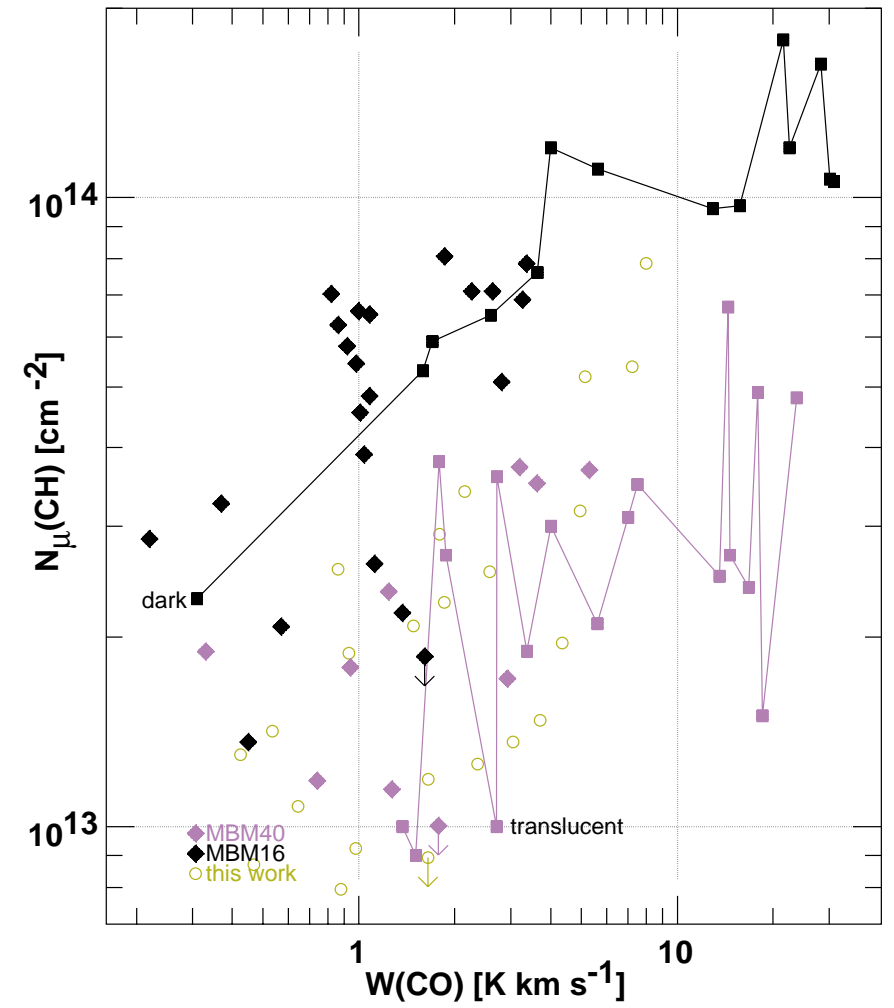

Fig. 7. Microwave-derived $\mathrm{CH}$ column densities $N_{\mu}(\mathrm{CH})$ and $\mathrm{CO}$ profile integrals $W(\mathrm{CO})$. The solid, connected symbols represent data tabulated by Magnani \& Onello (1995) for lines of sight considered "translucent" (shaded) and "dark" (shown dark). Mapping data for two high-latitude molecular clouds from Magnani et al. (1998) are shown as dark and shaded solid diamonds. Our data from Fig. 6 at bottom left have been overlaid as the open circles.

(nor did they note the bimodal behaviour which is clearly seen there). In passing we note that much of the data for so-called translucent gas was considered to sample diffuse material in the original reference (Federman \& Willson 1982).

The chained lines (connected solid symbols) in Fig. 7 show the $\mathrm{CH}$ and $\mathrm{CO}$ data tabulated by Magnani \& Onello (1995), manipulated only to the extent that one source considered translucent (3C353) has been reclassified as dark since an examination of the original reference (Federman et al. 1987) showed that a visual extinction of 6 mag was quoted for the position whose $N_{\mu}(\mathrm{CH})$ was used. The data from our Fig. 6 at lower left have been transferred to Fig. 7, and it is apparent that they overlap the "translucent" data of Magnani \& Onello (1995) except at the very highest values of the CO profile integral. Also shown in Fig. 7 is the more recent mapping data of Magnani et al. (1998) for two high-latitude clouds described as translucent. The observations for one of them, MBM40, all fall into the "translucent" regime; data for MBM 16 lie chiefly on the "dark" gas locus, except at the lowest values of $W(\mathrm{CO})$, and could be interpreted as showing an abrupt transition from diffuse to dark conditions at $W(\mathrm{CO})=1 \mathrm{~K} \mathrm{~km} \mathrm{~s}^{-1}$.

We can distinguish three modes of behaviour for the data in this figure. First, there is a slow increase of $N_{\mu}(\mathrm{CH})$ with $W(\mathrm{CO})$ for both the dark and diffuse/translucent gases: possible appearances aside, the best-fit power-law slope for the 
"dark" data of Magnani \& Onello (1995) has $N_{\mu}(\mathrm{CH}) \propto$ $W(\mathrm{CO})^{0.34 \pm 0.05}$ while $N_{\mu}(\mathrm{CH}) \propto W(\mathrm{CO})^{0.26 \pm 0.12}$ for the data along the line marked as "translucent." Second there is a nearly fixed, factor of 3 offset in $N_{\mu}(\mathrm{CH})$ between the dark cloud and diffuse/translucent material. The $\mathrm{CH}$ brightness appears bimodal at a given value of the $\mathrm{CO}$ profile integral in a variety of separate datasets and the offset in $N_{\mu}(\mathrm{CH})$ persists up to $\mathrm{CO}$ profile integrals at least as large as $20 \mathrm{~K} \mathrm{~km} \mathrm{~s}^{-1}$, which represents quite a strong $\mathrm{CO}$ line. For very small $\mathrm{CO}$ profile integrals $W(\mathrm{CO})<1 \mathrm{~K} \mathrm{~km} \mathrm{~s}^{-1}$, the dark and diffuse regimes may merge, which is intuitively understandable. The third discernible mode of behaviour is scatter in $N_{\mu}(\mathrm{CH})$ at a fixed $W(\mathrm{CO})$ which we ascribed above to variations in $\mathrm{CH}$ excitation and other local conditions, for the diffuse/translucent gas.

The strength of $\mathrm{CO}$ emission is expected to change rapidly in diffuse gas: $N\left(\mathrm{H}_{2}\right) \propto E_{B-V}{ }^{2}$ from Fig. 1, and $N(\mathrm{CO}) \propto$ $N(\mathrm{CH})^{2} \propto N\left(\mathrm{H}_{2}\right)^{2}$ (Federman \& Lambert 1988; Federman et al. 1994; Liszt \& Lucas 2000). So, it follows that the CH profile integral should vary much less rapidly than the $\mathrm{CO}$ brightness if $N_{\mu}(\mathrm{CH}) \propto N(\mathrm{CH})$. The change in $N\left(\mathrm{H}_{2}\right)$ across Fig. 7 should be smaller than that in $W(\mathrm{CO})$, for the diffuse gas at least, which would lead to a rather shallow slope. But it is far from obvious that the diffuse/translucent and dark gas components should have so nearly the same slope in their $N_{\mu}(\mathrm{CH})-$ $W(\mathrm{CO})$ relationship. Perhaps the shallowness of the slope in the dark gas reflects the decline of $X_{\mathrm{CH}}$ in denser gas, at higher $W(\mathrm{CO})$.

A literal interpretation of Fig. 7 implies that dark gas requires about 3 times as much $N\left(\mathrm{H}_{2}\right)$ to produce a given $W(\mathrm{CO})$ for $W(\mathrm{CO})=1-20 \mathrm{~K} \mathrm{~km} \mathrm{~s}^{-1}$ (this difference might be larger if $N_{\mu}(\mathrm{CH})$ is diminished in dark gas because $X_{\mathrm{CH}}$ is smaller). If such were the case, a substantial $\mathrm{CO}$ emission component from diffuse gas mixed into an ensemble of dark clouds could cause misestimation of $N(\mathrm{H})$. However, there is no other evidence for a diminished $N\left(\mathrm{H}_{2}\right) / W(\mathrm{CO})$ ratio in diffuse gas, and actual measurements, in the few possible cases, yield a typical value. For instance, from a comparison of the $\mathrm{CO}$ and $\mathrm{HCO}^{+}$ data in Figs. 12 and 13 of Liszt \& Lucas (1998), it follows that $N\left(\mathrm{H}_{2}\right) / W(\mathrm{CO}) \approx 2.5 \times 10^{20} \mathrm{H}_{2} \mathrm{~cm}^{-2} /\left(\mathrm{K} \mathrm{km} \mathrm{s}^{-1}\right)$ around $W(\mathrm{CO})=1-2 \mathrm{~K} \mathrm{~km} \mathrm{~s}^{-1}$. Toward $\zeta \mathrm{Oph}$, where all the relevant properties are directly measureable, we have $N\left(\mathrm{H}_{2}\right) / W(\mathrm{CO}) \approx$ $4 \times 10^{20} \mathrm{H}_{2} \mathrm{~cm}^{-2} /\left(\mathrm{K} \mathrm{km} \mathrm{s}^{-1}\right)($ Liszt 1982, 1997).

\subsection{Excitation}

Notably, the on-source $\mathrm{CH}$ profile is not weaker than the nearby off-source average in a single direction out of all those observed; the $\mathrm{CH} \Lambda$-doublet is generally inverted in diffuse gas. Figure 8 shows on and off-source spectra taken toward 3C111 and $3 \mathrm{C} 123$, the strongest continuum sources observed. Also shown are optical depth spectra made by applying Eq. (2) to the data taken on $\left(T_{\mathrm{bg}}=T_{\mathrm{cmb}}+T_{\mathrm{C}}\right)$ and off-source $\left(T_{\mathrm{bg}}=T_{\mathrm{cmb}}\right)$. In only one case (3C123) can we derive a statistically significant inversion $\left(T_{\text {exc }}<0\right)$. Even for $3 \mathrm{C} 111$, the excitation temperature derived from a further application of Eq. (2) (using the derived optical depth profile) is positive even where $\tau<0$ in

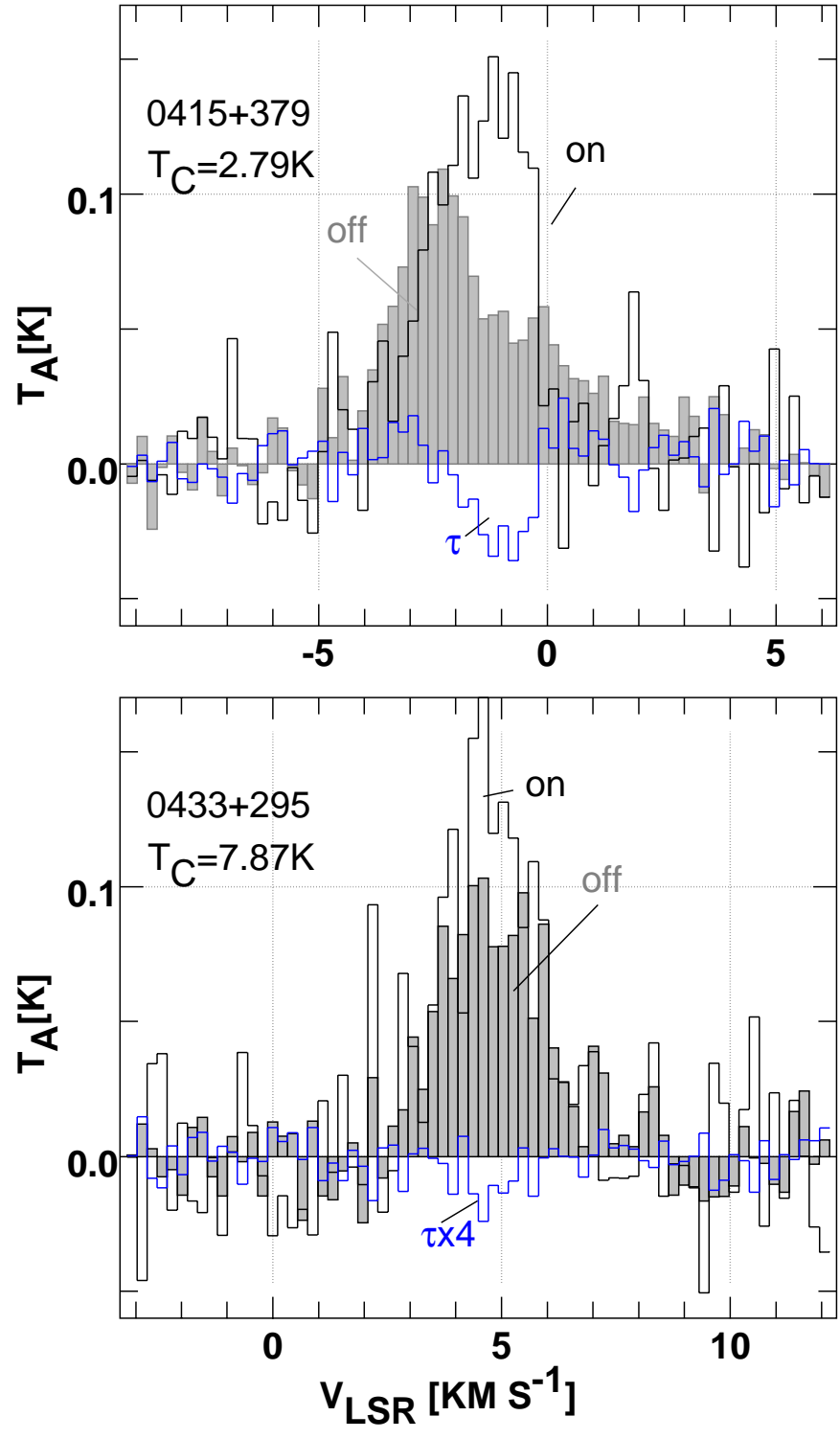

Fig. 8. On and off source spectra toward two strong continuum sources. The off-source average is shown shaded. An optical depth spectrum derived from Eq. (2) is shown in each case; for B0433+295 (3C123) $T_{\text {exc }}=-10.7 \pm 3.2$ for the five contiguous central channels showing $\tau<0$.

Fig. 8; spatial structure in the $\mathrm{CH}$ emission renders the solutions unreliable.

For $3 \mathrm{C} 123$, however, $\left\langle T_{\text {exc }}\right\rangle=-10.7 \pm 3.2 \mathrm{~K}$ averaged over the five contiguous central channels where $\tau<0$ in Fig. 8 and $-16<T_{\text {exc }}<-6 \mathrm{~K}$ over this same interval. This is essentially the same result as that obtained by Hjalmarson et al. (1977), $T_{\text {exc }}=-9 \pm 5 \mathrm{~K}$. Genzel et al. (1979) quote a value $T_{\text {exc }}=-60 \pm 30 \mathrm{~K}$ in this direction but the meaning of the (rather large) error or range is not clear from their presentation. Genzel et al. (1979) thus claim that the $3335 \mathrm{MHz}$ transition seen with the $100 \mathrm{~m}$ telescope is substantially less inverted than was found earlier using the Onsala $25 \mathrm{~m}$ dish. In the optically thin, Rayleigh-Jeans limit of the column density determination $N_{\mu}(\mathrm{CH}) \propto T_{\text {exc }} /\left(T_{\text {exc }}-T_{\text {cmb }}\right)$. The $T_{\text {exc }}$-dependent term in this expression is 0.96 for $T_{\mathrm{exc}}=-60 \mathrm{~K}, 0.85$ for $T_{\mathrm{exc}}=-15 \mathrm{~K}$, and 0.79 for $T_{\text {exc }}=-10 \mathrm{~K}$. 


\section{Summary}

Although fourth in the current series, this paper is actually also the third of three papers discussing singledish $\mathrm{cm}$-wave spectra of the molecules in diffuse gas, taken toward and around a sample of compact, extragalactic mm-wave continuum sources. In H I, such on-off comparison experiments have come to be known as emission-absorption experiments (Dickey et al. 1978) but as it turns out, we performed one emission-absorption experiment (in OH; Liszt \& Lucas 1996), one absorption-absorption experiment (in $\mathrm{H}_{2} \mathrm{CO}$, which appeared in absorption both on and off-source; Liszt \& Lucas 1995) and, here, one emission-emission experiment (since no true absorption was detectable).

We began by displaying the richly structured behaviour of $\mathrm{CH}$ with $E_{B-V} ; N(\mathrm{CH})$ is multi-valued with respect to $E_{B-V}$, depending on the degree of conversion to molecular gas along the line of sight, and a simple, linear $\mathrm{CH}-$ $E_{B-V}$ relationship can be expected only when the extinction is dominated by molecular gas, as toward a single dark cloud. Otherwise, the range of measured $N(\mathrm{CH})$ at a given $E_{B-V}$ in the diffuse gas as a whole is typically more than one order of magnitude. Much of this behaviour can be explained on the basis of an easily-demonstrated and long-known, nearly constant relative abundance $\left\langle X_{\mathrm{CH}}\right\rangle=4.3 \pm 1.9 \times 10^{-8}$, and $N(\mathrm{CH}) \propto N\left(\mathrm{H}_{2}\right)^{1.00 \pm 0.06}$ for $N\left(\mathrm{H}_{2}\right) \lesssim 10^{21} \mathrm{~cm}^{-2}$ : as well, we have that $N\left(\mathrm{H}_{2}\right) \propto E_{B-V}{ }^{1.8}$ for $0.2<E_{B-V}<0.7$.

If $\mathrm{CH}$ is a good predictor of $\mathrm{H}_{2}$, the 140 lines of sight gathered to study the $\mathrm{CH}-E_{B-V}$ relationship allow derivation of the molecular fraction in the diffuse/translucent ISM over a much wider range of sample mean densities $\left\langle E_{B-V}\right\rangle \mid\langle R\rangle$ than is directly accessible in measurements of the lines of hydrogen. The molecular fraction found in this way is in good agreement with direct measurements at low (Copernicus) and high (FUSE) sample mean density, and is $0.4-0.45$ for $\left\langle E_{B-V}>/<R>=0.61 \mathrm{mag} \mathrm{kpc}^{-1}\right.$, which is the accepted mean in the gas within 500-1000 pc.

We pointed out that sensitive optical measurements of the population ratio in the upper and lower halves of the groundstate $\mathrm{CH} \Lambda$-doublet toward two stars predict that the brightness of the microwave $\mathrm{CH}$ lines should be double-valued at a given $\mathrm{CH}$ column density in diffuse gas depending on whether the excitation is inverted (the brighter branch); this is consistent with models of $\mathrm{CH}$ excitation which predict a transition from normal excitation to inversion at hydrogen densites in the range $10-1000 \mathrm{~cm}^{-2}$, but the effect is not present in the microwave lines in these directions. This could be due to the disparity in beam-sizes or to relatively small errors in the optical data.

We presented $3335 \mathrm{MHz} \mathrm{CH}$ observations toward some of the compact extragalactic mm-wave continuum sources studied in this series of papers, toward two strong $\mathrm{cm}$-wave sources, and around $\zeta \mathrm{Oph}$, and compared the properties of $\mathrm{CH}$ with those of $\mathrm{OH}$ and $\mathrm{CO}$ in emission and $\mathrm{HCO}^{+}$and $\mathrm{C}_{2} \mathrm{H}$ seen in absorption. In stronger-lined gas, the $\mathrm{CH} / \mathrm{OH}$ comparison confirms the very small $\mathrm{OH}$ excitation temperatures which have been found in diffuse gas. Comparisons of $\mathrm{CH}$ with $\mathrm{HCO}^{+}$and $\mathrm{C}_{2} \mathrm{H}$ show that there is either a very large scatter in the $\mathrm{CH}$ brightness or microwave-derived $\mathrm{CH}$ column density at a given
$N\left(\mathrm{HCO}^{+}\right)$or $N\left(\mathrm{C}_{2} \mathrm{H}\right)$ or perhaps a bimodality. The $\mathrm{CH} / \mathrm{HCO}^{+}$ comparison readily (but only roughly) confirms our previouslyderived ratio $N\left(\mathrm{HCO}^{+}\right) / N\left(\mathrm{H}_{2}\right)=2 \times 10^{-9}$.

The $3335 \mathrm{MHz}$ line brightness in diffuse gas is very definitely bimodal with regard to $\mathrm{CO}$ emission in both the new data presented here and our previously-published data around $\zeta$ Oph. To explore this further, we compared our data with other published studies which used $\mathrm{CH}$ to derive the $\mathrm{CO}-\mathrm{H}_{2}$ conversion factor in diffuse/translucent gas and found that they are entirely consistent with our data. This may be a manifestation of a disparity between inverted and non-inverted $\mathrm{CH}$ expected in diffuse gas.

We found some hitherto-unnoticed systematic behaviour in the $\mathrm{CH}-\mathrm{CO}$ comparison in diffuse and dark gas, in particular a steady, factor of $\approx 3$ offset in the ratio of $\mathrm{CH}$ and $\mathrm{CO}$ profile integrals for $W(\mathrm{CO})=1-30 \mathrm{~K} \mathrm{~km} \mathrm{~s}^{-1}: W(\mathrm{CH}) / W(\mathrm{CO})$ is consistently larger by $\approx 3$ in dark gas. A shallow slope in the $W(\mathrm{CH})-W(\mathrm{CO})$ relationship in diffuse gas is undertandable because the $\mathrm{CO}$ abundance varies rapidly with $N\left(\mathrm{H}_{2}\right)$, $N(\mathrm{CO}) \propto N\left(\mathrm{H}_{2}\right)^{2}$ and the $\mathrm{CO}$ brightness will increase even faster than $N(\mathrm{CO})$, but the presence of nearly the same shallow slope $W(\mathrm{CH}) \propto W(\mathrm{CO})^{0.3}$ in dark and diffuse gas is puzzling. It may reflect the decline of $X_{\mathrm{CH}}$ which is known to occur in very dark gas.

The next paper in this series will discuss several species whose abundances are best determined at cm-wave frequencies, such as $\mathrm{NH}_{3}, \mathrm{H}_{2} \mathrm{CO}$ and $\mathrm{C}_{4} \mathrm{H}$.

Acknowledgements. The National Radio Astronomy Observatory is operated by AUI, Inc. under a cooperative agreement with the US National Science Foundation. IRAM is operated by CNRS (France), the MPG (Germany) and the IGN (Spain). The comments of the referee, John Black, were very helpful.

\section{References}

Allen, M. M. 1994, ApJ, 424, 754

Bertojo, M., Cheung, A. C., \& Townes, C. H. 1976, ApJ, 208, 914

Bohlin, R. C., Savage, B. D., \& Drake, J. F. 1978, ApJ, 224, 132

Bouloy, D., Nguyen-Q-Rieu, \& Field, D. 1984, A\&A, 130, 380

Bujarrabal, V., Salinas, F., \& Gonzalo, I. 1984, ApJ, 285, 312

Chaffee, F. H., \& Lutz, B. L. 1978, ApJ, 221, L91

Cox, P., Guesten, R., \& Henkel, C. 1988, A\&A, 206, 108

Crane, P., Lambert, D. L., \& Sheffer, Y. 1995, AJS, 99, 107

Crawford, I. A. 1995, MNRAS, 277, 458

Crawford, I. A., \& Williams, D. A. 1997, MNRAS, 291, L53

Crutcher, R. M. 1979, ApJ, 234, 881

Crutcher, R. M. 1985, ApJ, 288, 604

Danks, A. C., Federman, S. R., \& Lambert, D. L. 1984, A\&A, 130, 62

Dickey, J. M., Terzian, Y., \& Salpeter, E. E. 1978, AJ Suppl. Ser., 36,77

Federman, S. R. 1982, ApJ, 257, 125

Federman, S. R., Cardell, J. A., van Dishoeck, E. F., Lambert, D. L., \& Black, J. H. 1995, ApJ, 445, 325

Federman, S. R., Evans, N. J., Willson, R. F., et al. 1987, ApJ, 322, 960

Federman, S. R., \& Lambert, D. L. 1988, ApJ, 328, 777

Federman, S. R., Strom, C. J., Lambert, D. L., et al. 1994, ApJ, 424, 772

Federman, S. R., \& Willson, R. F. 1982, ApJ, 260, 124 


\section{Appendix A: Line profile integrals shown in Fig. 6}

Table A.1. Line profile integrals.

\begin{tabular}{lcccccc}
\hline \hline Source & $V$ & $\begin{array}{c}W(\mathrm{OH}) \\
\mathrm{K} \mathrm{km} \mathrm{s}^{-1}\end{array}$ & $\begin{array}{c}\int \tau\left(\mathrm{HCO}^{+}\right) \mathrm{d} v^{1} \\
\mathrm{~km} \mathrm{~s}^{-1}\end{array}$ & $\begin{array}{c}\int \tau\left(\mathrm{C}_{2} \mathrm{H}\right) \mathrm{d} v^{2} \\
\mathrm{~km} \mathrm{~s}^{-1}\end{array}$ & $\begin{array}{c}W(\mathrm{CO}) \\
\mathrm{K} \mathrm{km} \mathrm{s}^{-1}\end{array}$ & $\begin{array}{c}W(\mathrm{CH}) \\
\mathrm{K} \mathrm{km} \mathrm{s}^{-1}\end{array}$ \\
\hline B0212+735 & -10 & $0.0886(0.009)$ & $0.58(0.04)$ & $0.38(0.18)$ & $0.86(0.06)$ & $0.0719(0.01033)$ \\
B0212+735 & 0 & $<0.0134$ & $0.62(0.03)$ & $0.18(0.06)$ & $<0.07$ & $<0.01342$ \\
B0212+735 & 3 & $0.0889(0.0084)$ & $3.77(0.17)$ & $1.26(0.15)$ & $4.96(0.06)$ & $0.0906(0.00748)$ \\
B0415+379 & -2.2 & $0.4443(0.0106)$ & $11.91(0.334)$ & $1.31(0.03)$ & $8.00(0.07)$ & $0.2200(0.00389)$ \\
B0415+379 & -0.8 & $0.1614(0.0105)$ & $5.526(0.244)$ & $1.75(0.02)$ & $7.22(0.06)$ & $0.1506(0.0037)$ \\
B0433+295 & all & $0.4073(0.0157)$ & & & $0.2792(0.01294)$ \\
B0459+252 & all & $0.2251(0.0126)$ & & & $0.1835(0.00838)$ \\
B0528+134 & 2 & $0.0534(0.0098)$ & $0.2362(0.0103)$ & & $<0.13$ & $0.0563(0.01171)$ \\
B0528+134 & 10 & $0.1146(0.0097)$ & $1.836(0.019)$ & $0.39(0.05)$ & $2.15(0.06)$ & $0.0955(0.00961)$ \\
B0727-115 & all & & $0.476(0.052)$ & & & $<0.0191$ \\
B0736+017 & all & $0.0426(0.0101)$ & $0.802(0.063)$ & $0.40(0.04)$ & $0.88(0.05)$ & $0.0223(0.00813)$ \\
B0954+658 & all & $0.0442(0.0050)$ & $1.48(0.20)$ & $0.48(0.04)$ & $1.65(0.04)$ & $<0.025$ \\
B2013+370 & all & $0.3332(0.0310)$ & $1.785(0.0236)$ & & $5.15(0.23)$ & $0.1452(0.02359)$ \\
B2023+336 & all & $0.3185(0.0173)$ & & & $0.2009(0.0219)$ \\
B2200+420 & all & $0.0529(0.0066)$ & $2.36(0.03)$ & $1.15(0.20)$ & $5.78(0.05)$ & $<0.011$ \\
\hline
\end{tabular}

${ }^{1} N\left(\mathrm{HCO}^{+}\right)=1.02 \times 10^{12} \mathrm{~cm}^{-2} \int \tau\left(\mathrm{HCO}^{+}\right) \mathrm{d} v$.

${ }^{2} N\left(\mathrm{C}_{2} \mathrm{H}\right)=2.711 \times 10^{13} \mathrm{~cm}^{-2} \int \tau\left(\mathrm{C}_{2} \mathrm{H}\right) \mathrm{d} v$.

${ }^{3} \mathrm{HCO}^{+}$data toward $\mathrm{B} 0415+379$ are $60 \times N\left(\mathrm{H}^{13} \mathrm{CO}^{+}\right)$.

${ }^{4}$ Toward $3 \mathrm{C} 111$ components were separated by Gaussian fitting.

Felenbok, P., \& Roueff, E. 1996, ApJ, 465, L57

Genzel, R., Downes, D., Pauls, T., Wilson, T. L., \& Bieging, J. 1979, A\&A, 73, 253

Glassgold, A. E., \& Langer, W. D. 1975, ApJ, 197, 347

Glassgold, A. E., \& Langer, W. D. 1976, ApJ, 206, 85

Gredel, R., van Dishoeck, E. F., \& Black, J. H. 1993, A\&A, 269, 477

Hjalmarson, A., Sume, A., Elldér, J., et al. 1977, AJS, 35, 263

Jenniskens, P., Ehrenfreund, P., \& Desert, F.-X. 1992, A\&A, 265, L1

Joseph, C. L., Snow, T. P., Seab, C. G., \& Crutcher, R. M. 1986, ApJ, 309, 771

Jura, M., \& Meyer, D. M. 1985, ApJ, 294, 238

Lang, K. R., \& Willson, R. F. 1978, ApJ, 224, 125

Lien, D. J. 1984, ApJ, 284, 578

Liszt, H. S. 1982, ApJ, 262, 198

Liszt, H. S. 1997, A\&A, 322, 962

Liszt, H. S., \& Lucas, R. 1995, A\&A, 299, 847

Liszt, H. S., \& Lucas, R. 1996, A\&A, 314, 917

Liszt, H. S., \& Lucas, R. 1998, A\&A, 339, 561

Liszt, H. S., \& Lucas, R. 2000, A\&A, 355, 333

Liszt, H., \& Lucas, R. 2001, A\&A, 370, 576

Lucas, R., \& Liszt, H. S. 1998, A\&A, 337, 246

Lucas, R., \& Liszt, H. S. 1996, A\&A, 307, 237

Lucas, R., \& Liszt, H. S. 2000a, A\&A, 358, 1069

Lucas, R., \& Liszt, H. S. 2000b, A\&A, 355, 327

Lucas, R., \& Liszt, H. S. 2002, A\&A, 384, 1054

Münch, I. G. 1952, ApJ, 116, 575

Magnani, L., \& Onello, J. S. 1995, ApJ, 443, 169

Magnani, L., Onello, J. S., Adams, N. G., Hartmann, D., \& Thaddeus, P. 1998, ApJ, 504, 290
Mattila, K. 1986, A\&A, 160, 157

Meyer, D. M., \& Roth, K. C. 1991, ApJ, 376, L49

Nash, A. G. 1990, AJS, 72, 303

Ohishi, M., Irvine, W., \& Kaifu, N. 1992, in Astrochemistry of cosmic phenomena: proceedings of the 150th Symposium of the International Astronomical Union, held at Campos do Jordao, Sao Paulo, Brazil, August 5-9, 1991, ed. P. D. Singh (Dordrecht: Kluwer 1992), 171

Penprase, B. E. 1993, AJS, 88, 433

Rachford, B., Snow, T. P., Tumlinson, J., et al. 2002, ApJ, in press

Rachford, B. L., Snow, T. P., Tumlinson, J., et al. 2001, ApJ, 555, 839

Roueff, E. 1996, MNRAS, 279, L37

Rydbeck, O. E. H., Kollberg, E., Hjalmarson, A., et al. 1976, AJS, 31, 333

Sandell, G. 1982, in Regions of Recent Star Formation, ed. R. S. Roger, \& P. E. Dewdney, ASSL, 93, 479

Sandell, G., Johansson, L. E. B., Rieu, N. Q., \& Mattila, K. 1981, A\&A, 97, 317

Savage, B. D., Drake, J. F., Budich, W., \& Bohlin, R. C. 1977, ApJ, 216, 291

Snow, T. P., Rachford, B. L., Tumlinson, J., et al. 2000, ApJ, 538, L65

Spitzer, L. 1978, Physical processes in the interstellar medium (New York Wiley-Interscience), 333

Van Dishoeck, E. F., \& Black, J. H. 1986, AJS, 62, 109

van Dishoeck, E. F., \& Black, J. H. 1989, ApJ, 340, 273

Willson, R. F. 1981, ApJ, 247, 116 